\title{
Optimal Climate Policy When Damages are Unknown
}

\author{
Ivan Rudik*
}

Integrated assessment models (IAMs) are economists' primary tool for analyzing the optimal carbon tax. Damage functions, which link temperature to economic impacts, have come under fire because of their assumptions that may be incorrect in significant, but a priori unknowable ways. Here I develop recursive IAM frameworks to model uncertainty, learning, and concern for misspecification about damages. I decompose the carbon tax into channels capturing state uncertainty, insurance motives, and precautionary saving. Damage learning improves ex ante welfare by $\$ 750$ billion. If damage functions are misspecified and omit the potential for catastrophic damages, robust control may be beneficial ex post.

JEL: H23, Q54, Q58

Keywords: climate, integrated assessment, damages, deep uncertainty, robust control, learning, social cost of carbon, carbon tax

Integrated assessment models (IAMs) are economists' primary tool for analyzing the optimal carbon tax. IAMs are macroeconomic models linked to a climate module by the "damage function," which translates rising temperature into economic losses. ${ }^{1}$ The world's true underlying damage function

\footnotetext{
${ }^{*}$ Charles H. Dyson School of Applied Economics and Management, Cornell University, Ithaca, NY, USA, 14853-6201. irudik@cornell.edu. I am immensely grateful for advice and support from Derek Lemoine. I also benefited from comments and discussions with Lint Barrage, Price Fishback, Alex Hollingsworth, Dave Kelly, Stan Reynolds, and Quinn Weninger as well as participants at many seminars. Funding from The University of Arizona GPSC Research Grant is gratefully acknowledged.

${ }^{1}$ This is specific to the benchmark DICE model. Other IAMs may have explicit impacts of $\mathrm{CO}_{2}$. Despite varying levels of detail across IAMs, damage functional forms are largely uncertain and some economists have claimed they are implemented in IAMs in an ad hoc fashion (e.g. Pindyck, 2013).
} 
is complex and characterized by deep uncertainties stemming from a lack of knowledge of how warming will affect natural and economic systems. In order to develop tractable models in the face of these unknowns, integrated assessment modelers have historically pinned down the damage function with strong structural and parametric assumptions. Alongside the discount rate, the damage function is one of the most contentious feature of IAMs. In fact, some economists have suggested abandoning quantitative integrated assessment because of the alleged arbitrariness of the damage assumptions underpinning it (Pindyck, 2013, 2017).

Rather than abandoning the quantitative integrated assessment agenda that has proved useful for analysis and policymaking, I integrate uncertainty and even skepticism about damage functions into an IAM. I advance methodology in two ways. First, I incorporate parametric uncertainty about the parameters of the damage function and Bayesian learning about a parameter that maps into the temperature elasticity of damages. Second, I implement robust control, a technique to account for concerns from economists and scientists about structural damage function uncertainty, namely that it is misspecified in unknown ways. Using these methodological advances, I aim to ascertain the policy and welfare implications of: (1) accounting for parametric uncertainty over the damage function, (2) including endogenous damage learning over a parameter that has been a central focus of the climate economics literature, (3) allowing the policymaker to distrust the structure of her damage model via the use of robust control, and (4) interacting learning with the policymaker's distrust of her own model.

Similar to approaches in the saving literature (e.g., Gollier, 2004), I decompose the optimal carbon tax into different channels representing the policy implications of uncertainty and learning, and I present a novel channel representing concerns about model misspecification. The other uncertainty channels are analogous to precautionary saving and insurance motives and may increase or decrease the optimal carbon tax depending on both the current state of the world and whether the policymaker updates her damage beliefs. Without learning, the total effect of these uncertainty channels initially de- 
creases the carbon tax by a small amount, however over time it increases the carbon tax by up to 1 percent. With learning, the total effect always increases the carbon tax, by up to 5 percent. Insurance against model misspecification increases the optimal carbon tax by up to 5 percent when not learning, and slightly decreases the optimal carbon tax when learning.

Allowing for updating the estimate of the temperature elasticity of damages leads to ex ante present value welfare gains of about $\$ 750$ billion. Using robust control to guard against model misspecification increases the carbon tax over the next century, but if the model is correctly specified, these policy changes lead to welfare losses of $\$ 150$ billion. Since the purpose of robust control is to guard against unknown misspecifications I also compute ex post welfare gains when the damage function in the model is misspecified and the true damage function has an extra catastrophic term similar to Weitzman (2012) and Dietz and Stern (2015). Here I find that robust control can generate ex post present value welfare gains if catastrophe sets in at sufficiently low temperatures or $e x$ post welfare is calculated over a sufficiently long time horizon.

In this paper I develop an IAM by combining an annual version of the DICE model's economic system, a generalized version of the DICE damage function, and a reduced-form climate module that takes advantage of recent findings in climate science. Damage functions have been a central focus of analysis by researchers and have garnered a range of criticisms that may lead a policymaker to distrust IAMs. ${ }^{2}$ Broadly, economists have voiced their concerns about the calibration and parameterization of damage functions. Some researchers have suggested that the data used to calibrate damage functions are missing sectoral damage estimates such as diminished ecosystem services or increased healthcare costs (Howard, 2014), while others claim that the studies historically used in the calibration of damage functions underestimate the actual impact of warming (Hanemann, 2008; Howard and Sterner, 2017). The sharpest critiques focus on the functional form, saying that it is "...completely

\footnotetext{
${ }^{2}$ See Diaz and Moore (2017) for a review of damage function critiques, one of which is that there has not been sufficient attention paid towards parametric uncertainty and stochasticity. Both are accounted for here.
} 
ad hoc, with no theoretical or empirical foundation..." (Pindyck, 2013), and that there is "...no rationale, whether empirical or theoretical, for adopting [the DICE model's] quadratic form...although the practice is endemic in IAMs..." (Stanton, Ackerman and Kartha, 2009). Scientists and economists have said the damage function is far removed from evidence in economics and natural sciences (Ackerman and Stanton, 2012; Pindyck, 2013).

Damages functions in DICE and other IAMs often take a power functional form

$$
\text { Damages }=d_{1}[\text { Temperature }]^{d_{2}},
$$

where temperature is defined as the temperature increase over pre-industrial levels, damages are the percent loss of GDP, and where $d_{2}$, the temperature elasticity of damages, is assumed to be equal to 2 in DICE. The remaining parameter $d_{1}$ is calibrated by fitting the quadratic function to a set of the existing damage estimates. Some kind of aggregate damage functional form assumption is necessary for several reasons. First, optimizing climate policy requires us to be able to quantify the benefits of abating emissions, the avoided damages. Second, there has historically been a lack of theory and data to tell us what this damage functional form should be (Pindyck, 2013). Last, the ideal approach to capturing damages would be to develop a detailed competitive economy with a disaggregated set of sector- and location-specific damage functions, and with substitution and trade responses to climate damages. This is highly burdensome computationally, particularly if a modeler were to account for damage uncertainties.

Uncertainty and criticism about these key damage function parameters have lead to a proliferation of research aimed at understanding the sensitivity of policy prescriptions to parametric assumptions and the implications of parametric uncertainty. Recent work analyzing the sensitivity of optimal policy to both the functional form and calibration of the damage function has demonstrated that changing the damage function parameters may have nontrivial policy impacts (Stanton, Ackerman and Kartha, 2009; Kopp et al., 2012; Weitzman, 2012), and that several alternative polynomial functions can fit real world data just as well as the quadratic form (Stern, 2006; Hsiang 
et al., 2017). Because of the limitations of existing damage functions, some economists have developed alternative polynomial damage functions that better fit stylized facts about how we expect damages functions to act at high temperatures (Weitzman, 2010, 2012; Pindyck, 2012; Dietz and Stern, 2015), or explored alternative polynomial forms or coefficients as a sensitivity analysis (e.g. Dietz and Stern, 2015; Dietz and Venmans, 2019; Hsiang et al., 2017). ${ }^{3}$ Addressing these concerns about the damage function is critical since IAMs have become a key component of determining environmental regulation. For instance, a suite of IAMs has been used by the U.S. government to price greenhouse gas emissions in cost-benefit analyses of federal policies (Greenstone, Kopits and Wolverton, 2013).

The weaknesses of current damage functions and uncertainty about the economic consequences of additional warming indicate that damage uncertainty should be explicitly captured in modeling. Damage uncertainty has been included only recently in IAMs (Crost and Traeger, 2013, 2014), because the dynamic stochastic IAM literature is relatively new. The literature has heavily focused on policy and learning when the sensitivity of the climate to $\mathrm{CO}_{2}$ is uncertain (Kelly and Kolstad, 1999; Leach, 2007; Kelly and Tan, 2015; Fitzpatrick and Kelly, 2017; Hwang, Reynès and Tol, 2017; Lemoine and Rudik, 2017), even though there is evidence that damage uncertainty has more significant policy implications (Lemoine and McJeon, 2013). Dynamic stochastic IAMs have also recently been used to explore tipping points or irreversibilities (Lemoine and Traeger, 2014; Cai et al., 2015; Lontzek et al., 2015; Lemoine and Traeger, 2016a,b; Cai, Judd and Lontzek, 2018), and to examine the implications of geoengineering (Heutel, Moreno-Cruz and Shayegh, 2016, 2018). ${ }^{4}$

\footnotetext{
${ }^{3}$ Some of these papers use an exponential damage function however the exponential function maps into an infinite monomial series.

${ }^{4}$ The dynamic stochastic IAM literature expands on previous work that approximates uncertainty using Monte Carlo methods (Hope, 2006; Stern, 2006; Nordhaus, 2008; Ackerman, Stanton and Bueno, 2010; Kopp et al., 2012), however Monte Carlo analyses do not correctly capture decisionmaking under uncertainty (Lemoine and Rudik, 2017). There has been recent calls for improved treatment of risk, uncertainty, and ambiguity in pricing carbon (Stoerk, Wagner and Ward, 2018).
} 
Our current level of uncertainty in estimates of climate damages is not permanent. Uncertainty can be resolved through research, and in recent years, there has been an explosion of effort aimed at estimating sectoral climate damages. ${ }^{5}$ Moreover, there have also been recent surveys and meta-analyses aimed at updating estimates of aggregate global damage functions (Howard and Sylvan, 2016; Howard and Sterner, 2017). The updating of damage functions, and anticipation of future damage function updates by a forward-looking policymaker, has yet to be incorporated into an IAM.

Robust control and other techniques to account for model uncertainty have been recently applied environmental and integrated assessment contexts (Roseta-Palma and Xepapadeas, 2004; Athanassoglou and Xepapadeas, 2012; Anderson et al., 2014; Li, Narajabad and Temzelides, 2016; Berger, Emmerling and Tavoni, 2016). ${ }^{6}$ I build upon these strands of existing work by realistically capturing parametric uncertainty, endogenous learning and estimation of damages, and structural misspecification concerns in a recursive IAM where a policymaker trades off near-term costs of emissions reductions with the future benefits of lower temperature via reduced damages.

This paper makes several contributions to the broader discussion about the use of damage functions. First, if the standard damage functions in IAMs are misspecified and the true damage function follows one of the recently proposed catastrophic functional forms, ${ }^{7}$ protecting against model misspecification via robust control has limited welfare effects over the next 200 years relative to simply using a carbon tax optimized for a non-catastrophic damage function. This suggests that if real world climate policy eventually follows IAM policy prescriptions, guarding against these specific catastrophic misspecifications by

\footnotetext{
${ }^{5}$ See Hsiang (2016), Hsiang, Oliva and Walker (2019) and National Academy of Sciences (2017) for recent reviews of this literature. Hsiang et al. (2017) aggregates sectoral damage estimates in the United States and estimates a US-specific damage function. They also provide a framework for easily updating this damage function in response to new research.

${ }^{6}$ Robust control has also been applied to a policymaker learning about the dynamics of inflation and unemployment (Cogley et al., 2008).

${ }^{7}$ These catastrophic forms are informed by scientific arguments. For example, Weitzman (2012) and Dietz and Stern (2015) reference heat stress (Sherwood and Huber, 2010) and tipping points (Kriegler et al., 2009).
} 
using an even more aggressive carbon tax may not be of first-order importance. ${ }^{8}$ Second, when the true damage function has one of these catastrophic forms, updating a misspecified damage function can increase or decrease welfare by trillions of dollars depending on the true damage functional form and the time horizon for evaluating ex post welfare. This indicates that the realized social benefits of incorporating new damage estimates into IAMs will be highly dependent upon the damage functional form assumptions of these IAMs.

The paper is organized as follows. Section I gives more background on the construction of the damage function, and provides an overview of the dynamic stochastic IAM and all four frameworks. Section II describes how to decompose the optimal carbon tax under uncertainty into different channels. Section III reports results on how the different frameworks affect optimal policy and welfare. Section IV concludes. The appendix fully describes the IAM in this paper, details the computational methodology, provides a numerical error analysis, and provides a full derivation of the optimal carbon tax and its decomposition.

\section{The Damage Function and the Integrated Assessment Modeling Framework}

I begin by giving a brief overview of the DICE damage function, which is the foundation for the damage function in this paper. Next, I outline the IAM as a whole and then describe the four frameworks I use to investigate damage uncertainty, learning, and misspecification concerns through the use of robust control.

\footnotetext{
${ }^{8}$ If climate sensitivity was uncertain as in Kelly and Kolstad (1999), Kelly and Tan (2015), or Lemoine and Rudik (2017) then a particularly sensitive climate may result in extreme warming and catastrophic damages. In this case there may be large ex post benefits from robust control. Here I do not explore uncertainty about the climate sensitivity but interactions between these two uncertainties is important and should be studied in future work.
} 


\section{I.A The DICE Damage Function}

In the benchmark DICE model, time $t+1$ damages, $D\left(T_{t+1}^{s}\right)$, multiplicatively reduce the level of time $t+1$ output, $Y_{t+1}^{g}$, as a function of the time $t+1$ surface temperature, $T_{t+1}^{s} \cdot{ }^{9}$ The damage function specific to the DICE model is given by

$$
D\left(T_{t+1}^{s}\right)=d_{1}\left[T_{t+1}^{s}\right]^{d_{2}},
$$

where $d_{2}$ is assumed to be 2 and $d_{1}$ is calibrated to estimates of damages from specific levels of warming over preindustrial levels.

The most recent version of the DICE damage function is directly calibrated to a set of monetized damage estimates contained in Tol (2009), with an upward adjustment of 25 percent to account for impacts that are more difficult to estimate such as human conflict (Nordhaus and Sztorc, 2013). Even with this change in the damage calibration procedure, it does not include damage estimates from a burgeoning strand of literature that has estimated climate damages in a variety of sectors. ${ }^{10}$

\section{I.B Four Frameworks for Damage Uncertainty and The Dynamic Programming DICE Model}

In light of critiques about the damage function, I develop four different frameworks for investigating the policy and welfare implications of damage uncertainty. The uncertainty framework has the policymaker applying distributions over $d_{1}$ and $d_{2}$ and treating these parameters as uncertain. The learning framework allows the policymaker to learn and refine her beliefs about $d_{2}$ over time. The robust control framework has the policymaker being uncertain about both

\footnotetext{
${ }^{9}$ Although not investigated here, there are models that incorporate damages directly on capital (Kopp et al., 2012; Dietz and Stern, 2015) or utility (Sterner and Persson, 2008; Barrage, Forthcoming). Sterner and Persson (2008) explicitly include non-market environmental services in the utility function with limited substitutability, which can be degraded by warming. There is also empirical evidence that climate damages affect the growth rate of output, not just the level (Dell, Jones and Olken, 2012), and this may have substantial policy impacts (Moore and Diaz, 2015).

${ }^{10}$ See Hsiang (2016) for references to several recent examples.
} 


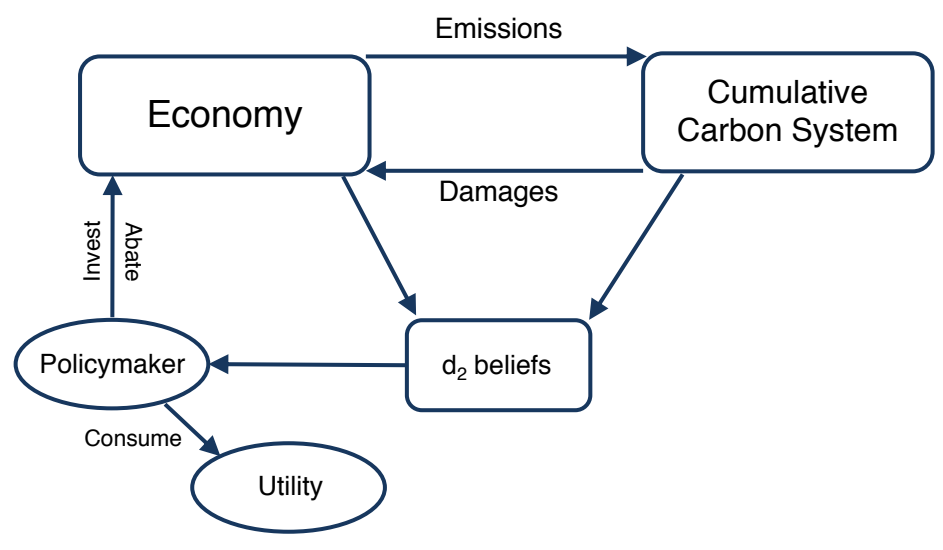

Figure 1: A schematic of this paper's IAM with learning. Capital, labor and technology are combined to produce output. Emissions from factor production flow into the cumulative carbon framework representation of the joint carbonclimate system. Greater cumulative emissions raise surface temperature which damages output. A learning policymaker uses her observations of temperature and realized damages to refine her beliefs. Her beliefs influence her policy choices.

parameters, but the framework also applies robust control to capture concerns that the damage function in the model is misspecified. Finally, the robust control and learning $(R C+L)$ framework has the policymaker combine updating her beliefs about $d_{2}$ with the use of robust control.

The IAM I use to study damage uncertainty is a finite-horizon, annual timestep Ramsey-Cass-Koopmans growth model coupled to a cumulative carbon framework representation of the joint carbon-climate system. ${ }^{11}$ The state space is composed of capital; the cumulative emissions of $\mathrm{CO}_{2}$; a state to capture the realized amount of damages; and states for the location and scale parameters governing the distribution of the policymaker's beliefs about $d_{2}$.

Figure 1 displays a schematic of the model. The full model description can be found in the online appendix. The model begins in 2005 and ends in 2605

\footnotetext{
${ }^{11}$ This representation of the climate system has recently been gaining traction in climate economics (Anderson et al., 2014; Berger and Marinacci, 2017; Brock and Xepapadeas, 2017; Dietz and Venmans, 2019).
} 
with a given terminal value function. ${ }^{12}$ Each period, the economy begins with an existing level of capital, labor and technology. These are combined in a Cobb-Douglas production function to generate output.

The production process generates $\mathrm{CO}_{2}$ emissions, and the cumulative amount of $\mathrm{CO}_{2}$ emitted is stored as a state variable. IAMs like DICE often use a complex multi-state carbon-climate systems to model climate dynamics. However recent advances in climate science have indicated that surface temperature $T_{t+1}^{s}$ is proportional to the cumulative amount of $\mathrm{CO}_{2}$ emissions $E_{t+1}$, both in the historical data and over different global climate model simulations spanning multiple climate scenarios and timeframes (Matthews et al., 2009; IPCC, 2013; Knutti, Rugenstein and Hegerl, 2017). ${ }^{13}$ Exploiting this reduced-form relationship, surface temperature is then given by

$$
T_{t+1}^{s}=\zeta E_{t+1}
$$

where $\zeta$ is called the transient climate response to emissions. Higher surface temperature causes more damage and reduces output through the damage function.

The remaining output after damages can be used in three ways: investment to increase the future stock of capital, abatement to reduce industrial $\mathrm{CO}_{2}$ emissions caused by factor production, or consumption to increase flow utility. The policymaker's objective is to maximize her discounted expected stream of utility from consumption. When deciding on the optimal way to divide output between abatement, consumption, and investment, the policymaker uses her current beliefs about future damages. These beliefs are given by probability distributions over the damage function parameters $d_{1}$ and $d_{2}$, and the probability distribution over a multiplicative damage shock $\omega_{t+1}$ that inhibits her learning about $d_{2}$. If the policymaker learns over time, she uses

\footnotetext{
${ }^{12}$ The same terminal value function is used across all four frameworks since it is sufficiently far in the future to have a negligible impact on policy. For example, given the growthadjusted discount factor in the model, $\$ 1$ at the terminal time 2605 is discounted to far less than $\$ 0.01$ in 2105 .

${ }^{13}$ The online appendix contains more details on why this relationship arises.
} 


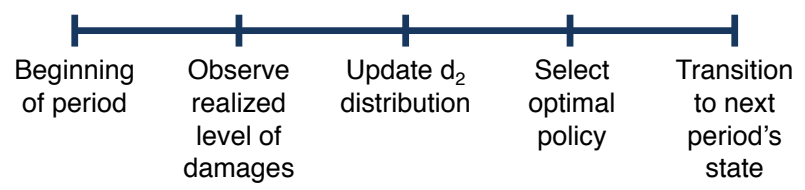

Figure 2: The timeline for each period $t$. The policymaker begins by observing the realization of damages which was unknown prior to the beginning of the period due to uncertainty about the damage function parameters and damage shock. She then uses the damage observation to update her beliefs about $d_{2}$. Finally, she optimizes her policy conditional on the current state of the world which includes capital, $\mathrm{CO}_{2}$, the amount of damages, and the parameters governing her updated beliefs.

observations of surface temperature (or equivalently cumulative emissions) and damages in a given period to update her beliefs about $d_{2}$.

The timing of the model is shown in Figure 2. The period begins and then the policymaker observes the state of the world. Damages are unknown prior to the beginning of the period because the policymaker does not know the true value of the damage parameters and because of objective stochasticity in the realization of the damage shock. After the policymaker observes the current state, she uses Bayes' Law to update her distribution over $d_{2}$. Next, with her new beliefs, she selects levels of abatement, consumption, and investment that maximize her expected welfare. Finally, the world transitions to the next period.

The policymaker's problem can be represented by a Bellman equation

$$
V_{t}\left(\boldsymbol{S}_{\boldsymbol{t}}\right)=\max _{c_{t}, \alpha_{t}}\left\{U\left(c_{t}\right)+\beta_{t} \mathbb{E}_{d_{2}, d_{1}, \omega_{t+1}}\left[V_{t+1}\left(\boldsymbol{S}_{\boldsymbol{t + 1}}\right)\right]\right\}
$$

subject to:

$$
\boldsymbol{S}_{t+1}=f\left(\boldsymbol{S}_{t}, c_{t}, \alpha_{t}\right)
$$

where $\boldsymbol{S}_{\boldsymbol{t}}$ is the time $t$ state vector, $\alpha_{t}$ is abatement, $c_{t}$ is consumption, $U\left(c_{t}\right)$ is her flow utility function, $V_{t}\left(\boldsymbol{S}_{\boldsymbol{t}}\right)$ is the policymaker's time $t$ value function, $f$ is the set of state transition equations, $\beta_{t}$ is a growth-adjusted discount factor, and $\mathbb{E}$ is the expectation operator. Expectations are always taken using the 
time $t$ information set. In each period, the policymaker maximizes the sum of her current flow utility and her discounted expected continuation value where she takes expectations over the two damage function parameters and the damage shock using her time $t$ beliefs. The appendix contains the full representation of the policymaker's problem.

\section{I.C The Damage Function Calibration and Learning}

In all four frameworks the policymaker takes both parameters of the damage function as uncertain. At time $t$ the policymaker assigns the damage coefficient a lognormal prior distribution, $d_{1} \sim \log \mathcal{N}\left(\mu_{c}, \sigma_{c}^{2}\right)$ and she assigns the damage exponent a normal distribution, $d_{2} \sim \mathcal{N}\left(\mu_{t}^{e}, \Sigma_{t}^{e}\right)$. In the frameworks with learning, the policymaker updates her distribution over $d_{2}$ each period. There are no time subscripts on the $d_{1}$ prior parameters since the policymaker is not learning about $d_{1}$. I select these distributional families since they admit Markovian updating rules which makes modeling learning tractable. ${ }^{14}$ The policymaker's immediate learning of the value of $d_{2}$ is hindered by a sequence of independent and identically distributed lognormal random damage shocks. These shocks capture random variation in how warming affects factor production through, for example, random variation in the frequency and magnitude of droughts or cyclones. The shock $\omega_{t+1}$ enters the damage function multiplicatively

$$
D\left(T_{t+1}^{s}, \omega_{t+1}\right)=d_{1}\left(T_{t+1}^{s}\right)^{d_{2}} \omega_{t+1} .
$$

Here I focus on time $t+1$ damages so the timing matches the carbon tax derivation in Section II. $Y_{t+1}^{g}$ is the gross output from factor production prior to any damages, and $Y_{t+1}^{n}$ is net output after damages. Damages reduce the

\footnotetext{
${ }^{14}$ Tractable learning has played a role in distributional family selection in the prior literature (e.g. Kelly and Kolstad, 1999; Kelly and Tan, 2015; Lemoine and Rudik, 2017).
} 
level of gross output in the following fashion (Nordhaus, 2008)

$$
Y_{t+1}^{n}=\frac{Y_{t+1}^{g}}{1+d_{1}\left(T_{t+1}^{s}\right)^{d_{2}} \omega_{t+1}}
$$

Rearranging the equation and taking the natural logarithm of both sides yields an equation such that observed variables are on the left hand side, and all unobserved variables are on the right hand side

$$
\log \left(\frac{Y_{t+1}^{g}}{Y_{t+1}^{n}}-1\right)=\log \left(d_{1}\right)+d_{2} \log \left(T_{t+1}^{s}\right)+\log \left(\omega_{t+1}\right)
$$

When the policymaker begins time period $t+1$, she observes the stochastically evolving level of output net of damages, $Y_{t+1}^{n}$, and she infers gross output before damages, $Y_{t+1}^{g}$, using the production function, the level of technology, and the capital and labor stocks. ${ }^{15}$ This comprises all the variables on the left hand side. On the other side of the equation, $\log \left(d_{1}\right), d_{2}$, and $\log \left(\omega_{t+1}\right)$ are normally distributed random variables that are not directly observed, while $\log \left(T_{t+1}^{s}\right)$ is observed by the policymaker.

Define $Q_{t+1} \equiv \log \left(\frac{Y_{t+1}^{g}}{Y_{t+1}^{n}}-1\right)$. Since the random variables on the right hand side of equation (3) are linearly separable, we have that

$$
Q_{t+1} \sim \mathcal{N}\left(\mu_{c}+\mu_{t+1}^{e} \log \left(T_{t+1}^{s}\right)+\mu_{\omega}, \sigma_{c}^{2}+\Sigma_{t}^{e}\left[\log \left(T_{t+1}^{s}\right)\right]^{2}+\sigma_{\omega}^{2}\right)
$$

where $\mu_{\omega}$ and $\sigma_{\omega}^{2}$ are the location and scale parameters of the damage shock $\omega_{t+1} \cdot{ }^{16}$ With each observation of the random variable $Q_{t+1}$ equal to some realized value $q_{t+1}$ at time $t+1$, the learning policymaker updates the parameters

\footnotetext{
${ }^{15}$ This is an approximation to the real world learning process. In reality we observe net output, but we may not know the global production function nor stock of capital that we required to back out gross output. However, researchers have been estimating damages over time in different sectors using observations of temperature fluctuations and measures of production. See Hsiang (2016) for examples of recent research.

${ }^{16}$ Since $d_{1}$ is not being learned and the parameters of its distribution are constant, $d_{1} \omega_{t+1}$ could be combined into a single random variable $d_{1} \omega_{t+1} \equiv \tilde{\omega}_{t+1} \sim \log \mathcal{N}\left(\mu_{c}+\mu_{\omega}, \sigma_{c}^{2}+\sigma_{\omega}^{2}\right)$.
} 
of her prior over $d_{2}$ according to Bayes' Law

$$
\begin{gathered}
\mu_{t+1}^{e}=\frac{\left(\sigma_{\omega}^{2}+\sigma_{c}^{2}\right) \mu_{t}^{e}+\log \left(T_{t+1}^{s}\right) \Sigma_{t}^{e}\left[q_{t+1}-\left(\mu_{c}+\mu_{\omega}\right)\right]}{\left(\sigma_{\omega}^{2}+\sigma_{c}^{2}\right)+\left[\log \left(T_{t+1}^{s}\right)\right]^{2} \Sigma_{t}^{e}} \\
\Sigma_{t+1}^{e}=\frac{\Sigma_{t}^{e}\left(\sigma_{\omega}^{2}+\sigma_{c}^{2}\right)}{\left(\sigma_{\omega}^{2}+\sigma_{c}^{2}\right)+\left[\log \left(T_{t+1}^{s}\right)\right]^{2} \Sigma_{t}^{e}}
\end{gathered}
$$

$\mu_{t+1}^{e}$, the location parameter and mean of the policymaker's beliefs about $d_{2}$, is a weighted average of its previous value, and the realization of $q_{t+1}-\left(\mu_{c}+\mu_{\omega}\right)$, a noisy signal of the true value of $d_{2}$. In expectation it is equal to $\mu_{t}^{e}$. $\Sigma_{t+1}^{e}$, is the scale parameter and variance of the policymaker's beliefs about $d_{2}$.

I estimate the distributions over $d_{1}, d_{2}$, and $\omega_{t+1}$ using data on temperature and damages from the recent meta-analysis by Howard and Sterner (2017). ${ }^{17}$ Specifically I estimate the logarithm of the damage function definition in equation $(2)$

$$
\log \left(\text { Damages }_{i}\right)=\log \left(d_{1}\right)+d_{2} \log \left(\text { Temperature }_{i}\right)+\varepsilon_{i}
$$

using ordinary least squares where $i$ indicates a separate estimate within the meta-analysis. ${ }^{18}$ The estimate on the $\log$ temperature coefficient $\hat{d}_{2}$ is distributed $\mathcal{N}\left(\mu_{0}^{e}, \Sigma_{0}^{e}\right)$ where $\mu_{2005}^{e}$ and $\Sigma_{2005}^{e}$ are the point estimate and squared standard error, and the subscript 2005 indicates these will be the values for the policymaker's initial beliefs in 2005. $\hat{d}_{2}$ has the conventional regression interpretation as the estimate of the temperature elasticity of damages. Similarly, $\left.\log \hat{(} d_{1}\right) \sim \mathcal{N}\left(\mu_{c}, \sigma_{c}^{2}\right)$ yields the estimated distribution of $\log \left(d_{1}\right)$, or if this term is exponentiated we have that $d_{1} \sim \log \mathcal{N}\left(\mu_{c}, \sigma_{c}^{2}\right)$. Finally the estimated variance for the disturbance term $\varepsilon_{i}$ yields the variance of $\log \left(\omega_{t+1}\right)$.

Table 1 shows the estimates for the location and scale parameters of the three distributions. The location $\left(\mu_{c}\right)$ and scale $\left(\sigma_{c}^{2}\right)$ parameters for $d_{1}$ imply an expectation of 0.00556 and a standard deviation of 0.00379 . The expected coefficient is approximately double that of the standard DICE calibration.

\footnotetext{
${ }^{17}$ The dataset includes estimates of severe and catastrophic damages (Weitzman, 2010; Burke, Hsiang and Miguel, 2015). The temperature data are in terms of anomalies and the damage data are in percentage terms.

${ }^{18}$ Taking the logarithm allows me to estimate the parameters with a linear regression.
} 
Table 1: Estimates for the location and scale parameters of the lognormally distributed damage coefficient, normally distributed damage exponent, and lognormally distributed damage shock.

\begin{tabular}{lcccccccc}
\hline & \multicolumn{2}{c}{ Coefficient } & & \multicolumn{2}{c}{ Exponent } & & \multicolumn{2}{c}{ Shock } \\
\cline { 2 - 3 } \cline { 8 - 9 } Parameter & $\mu_{c}$ & $\sigma_{c}^{2}$ & & $\mu_{2005}^{e}$ & $\Sigma_{2005}^{e}$ & & $\mu_{\omega}$ & $\sigma_{\omega}^{2}$ \\
Estimated Value & -5.31 & 0.23 & & 1.88 & 0.203 & & -0.59 & 1.18 \\
\hline
\end{tabular}
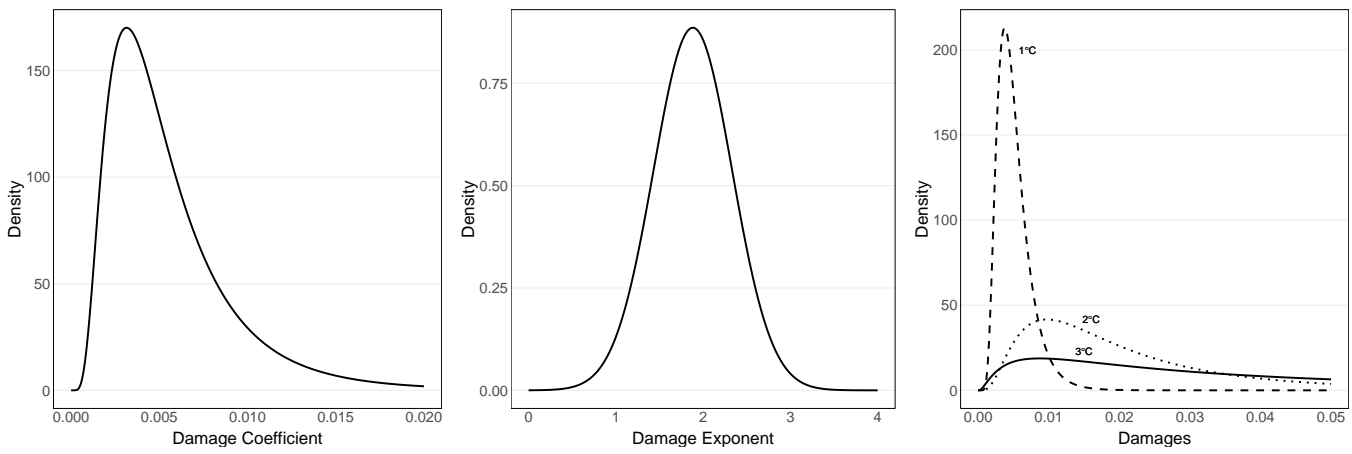

Figure 3: The probability density function of the coefficient's distribution (left), the probability density function of the exponent's prior distribution in 2005 (middle), the probability density function of damages given 2005 beliefs at $1^{\circ} \mathrm{C}, 2^{\circ} \mathrm{C}$, and $3^{\circ} \mathrm{C}$ of warming (right).

The mean estimate for $d_{2}$ is approximately equal the the selected value of 2 in DICE. The damage shock $\omega_{t+1}$ has mean of 1 and a standard deviation of 1.49 .

The year 2005 distribution for $d_{1}$ is shown in the left panel of Figure 3, and the year 2005 distribution for $d_{2}$ is shown in the center panel. The right panel of Figure 3 displays the distribution of damages at $1^{\circ} \mathrm{C}, 2^{\circ} \mathrm{C}$, and $3^{\circ} \mathrm{C}$ using the year 2005 information set. The $1^{\circ} \mathrm{C}$ distribution is highly peaked at damages of less than 1 percent of output, and the right tail decays rapidly. The $2{ }^{\circ} \mathrm{C}$ and $3^{\circ} \mathrm{C}$ distributions peak at slightly higher damages, but are characterized by right tails that decay more slowly and assign greater weight to higher damage outcomes. 


\section{I.D Robust Control}

Ideally, skepticism about damage functions should be built into an IAM by modeling a policymaker who does not completely trust that the damage function in her model is a precise representation of reality. In this case, the policymaker would believe that her damage model is only an approximation to the real world, and she would recognize that the optimal policy she obtains from her approximating model will almost surely not be optimal if taken and applied in real world policy. Instead of striving to develop an optimal policy using a model that is likely incorrect, the policymaker can instead try to find policy rules that are robust to unknown, and in the short-term unlearnable, errors in her model. ${ }^{19}$

To capture concerns about damage function misspecifications, I model a policymaker who uses robust control techniques in order to find policies that perform well even when the damage function inside her IAM may be incorrect (Hansen and Sargent, 2007, 2008). The policymaker begins with her approximating model in equation (2) and the associated parameter distributions. She then optimizes her policy, but she does not optimize policy conditional on only her approximating model. She instead optimizes over a set potential models that are close to her approximating model in terms of Kullback-Leibler divergence. The policymaker believes the true model likely resides in this set, but because the models in this set are all relatively similar to her own, they are difficult to statistically distinguish from one another over the time frame of her policymaking. During policy optimization, alternative damage models more similar to her approximating model are given greater weight, while those that are more different get less weight. By taking this approach the policymaker is recognizing that her approximating damage function is not exactly correct,

\footnotetext{
${ }^{19}$ Implicitly the four frameworks are taking different approaches to estimating an unknown data generating process for damages. In the uncertainty framework a monomial damage function is estimated once before policymaking begins. The learning framework allows for periodic updates to the model, the robust control framework considers the possibility of other approximations to the damage function, and the framework combining robust control and learning allows for the possibility of other damage functions with continual re-estimation of the exponent on the approximating damage function.
} 
and she wishes to guard against unknown errors. ${ }^{20}$

Omitting the transition equations, the policymaker's objective under robust control can be formulated as a min-max problem (Hansen and Sargent, 2008)

$V_{t}\left(\boldsymbol{S}_{\boldsymbol{t}}\right)=\min _{q_{t+1}}\left\{\max _{c_{t}, \alpha_{t}}\left[u\left(c_{t}\right)+\beta_{t}\left(\int \theta D_{t+1}\left(p_{t+1} \| q_{t+1}\right)+V_{t+1}\left(\boldsymbol{S}_{\boldsymbol{t + 1}}\right) d q_{t+1}\right)\right]\right\}$

where $\theta \in(0, \infty]$ is called the penalty parameter and will be described below, $D_{t+1}\left(p_{t+1} \| q_{t+1}\right)$ is the Kullback-Leibler divergence function

$$
D_{t+1}\left(p_{t+1} \| q_{t+1}\right)=\int \log \left(\frac{d p_{t+1}}{d q_{t+1}}\right) d p_{t+1}
$$

and $p_{t+1}$ is her approximating model's probability measure for taking expectations. $q_{t+1}$ is a distorted probability measure that she acts as though is the true model when she selects her controls, however $p_{t+1}$ still governs the actual state transitions. In other words, $q_{t+1}$ only affects the policymaker's decision rule, not the actual state transitions.

Hansen and Sargent (2008) provide an intuitive interpretation of the robust control formulation in equation (7). $q_{t+1}$ is the optimal selection of a probability measure by an evil agent whose objective is to minimize the payoff of the policymaker. The evil agent selects a new $q_{t+1}$ at each time $t$. The evil agent's selection of $q_{t+1}$ is penalized proportionally to the Kullback-Leibler divergence of its chosen distorted probability measure $q_{t+1}$ relative to the approximating model's probability measure $p_{t+1}$, where the coefficient of proportionality of the penalty is $\theta$, the penalty parameter. Large values of $\theta$ significantly penalize the evil agent for selecting probability measures $q_{t+1}$ that are much different than the approximating model $p_{t+1}$, while small values of $\theta$ let the evil agent select large distortions without much cost.

The distortions by the evil agent are what induce robust decision rules

\footnotetext{
${ }^{20}$ This is in contrast to maxmin expected utility which maximizes welfare subject to the worst-case model in some set (Gilboa and Schmeidler, 1989).
} 
on behalf of the policymaker. Since the evil agent aims to reduce the policymaker's welfare, the distortions it selects skew the policymaker's perceived expected continuation value downward. This induces her to select policies that are designed for lower welfare worlds, i.e. worlds where the damage function is more severe. By implicitly changing the policymaker's beliefs about the damage function to be more pessimistic, it induces her to uptake more precautionary action. How strongly she guards against misspecifications is inversely related to the size of $\theta$ since it controls the cost of the evil agent selecting a more distorted model $q_{t+1}$.

The policymaker maximizes her objective while accounting for potential distortions to her approximating model's probability measure, weighted by their Kullback-Leibler divergence. Equation (7) is equivalent to replacing the continuation value in the policymaker's Bellman equation in equation (1) with a risk sensitivity operator $T^{1}$ (Hansen and Sargent, 2007) that is tractable to use in dynamic programming settings ${ }^{21}$

$$
T^{1}\left(\beta_{t} V_{t+1}\left(\boldsymbol{S}_{\boldsymbol{t + 1}}\right) \mid \theta\right)=-\theta \log \left(\mathbb{E}_{d_{1}, \omega_{t+1}}\left[\exp \left(-\frac{\beta_{t} V_{t+1}\left(\boldsymbol{S}_{\boldsymbol{t + 1}}\right)}{\theta}\right)\right]\right),
$$

where the expectation operator is over $d_{1}$ and $\omega_{t+1}$. This results in a new Bellman equation

$$
V_{t}\left(\boldsymbol{S}_{\boldsymbol{t}}\right)=\max _{c_{t}, \alpha_{t}}\left\{U\left(c_{t}\right)+\mathbb{E}_{d_{2}}\left[T^{1}\left(\beta_{t} V_{t+1}\left(\boldsymbol{S}_{\boldsymbol{t + 1}}\right) \mid \theta\right)\right]\right\}
$$

subject to:

$$
\boldsymbol{S}_{t+1}=f\left(\boldsymbol{S}_{t}, c_{t}, \alpha_{t}\right)
$$

where the expectation acting outside the risk sensitivity operator is over $d_{2} .{ }^{22}$

\footnotetext{
${ }^{21}$ The distortions induced by the risk sensitivity operator are equivalent to the policymaker facing state-dependent shocks to the transition distributions, so that the distortions can be temporally linked and persist over time. This is what allows the distortions to represent real misspecifications to the policymaker's model.

${ }^{22}$ Since the risk sensitivity operator is acting directly on the time-invariant distributions of $d_{1}$ and $\omega_{t+1}$, learning about $d_{2}$ will not result in complete dissipation of the effects of robust control, although changing beliefs about $d_{2}$ may affect how the risk sensitivity operator alters the policymaker's decisions.
} 


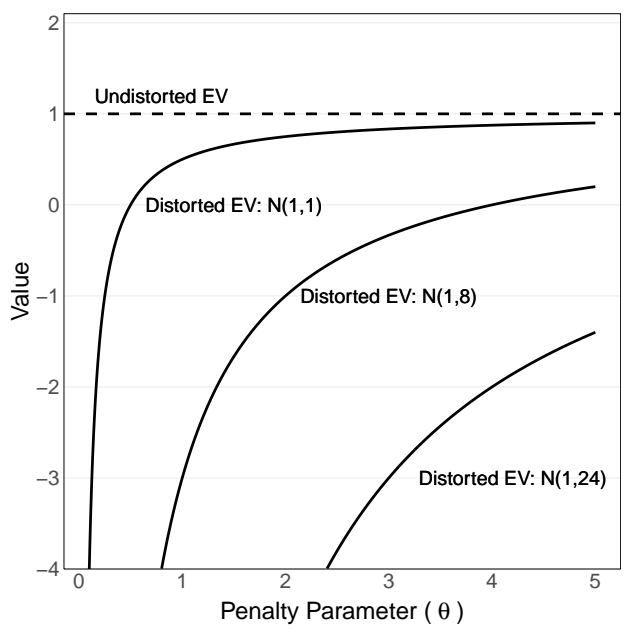

Figure 4: Three examples of the risk sensitivity operator distorting distributions. The plot displays the distorted expected value of three normal distributions as a function of the the penalty parameter. All three distributions have mean 1 , but have variances equal to 1 (top), 8 (middle), or 24 (bottom). The undistorted expected value is the dashed line and is independent of the penalty parameter.

All expectations are taken using the time $t$ information set.

Figure 4 shows three simple cases to illustrate how the risk sensitivity operator distorts the policymaker's expected continuation value. Consider a case where the continuation value, $V_{t+1}$, has a normal distribution with mean equal to 1 , and variance equal to 1,8 , or 24 . For simplicity, let the discount factor $\beta_{t}=1$ and assume $d_{2}$ is known so we can ignore the expectation over $d_{2}$ outside the risk sensitivity operator.

When we apply the risk sensitivity operator to $V_{t+1}$, the exponential term inside the expectation over $d_{1}$ and $\omega_{t+1}$ has a lognormal distribution which allows us to obtain a closed form solution for the expected continuation value: $1-\frac{\sigma^{2}}{2 \theta}$ where $\sigma^{2}$ is the variance. ${ }^{23}$ Figure 4 plots these distorted continuation values as a function of the penalty parameter and illustrates two key points. First, a smaller $\theta$ allows the evil agent to select transition probability measures that are increasingly distorted: for any given distribution, a smaller $\theta$ results

\footnotetext{
${ }^{23}$ The general takeaways will hold for other types of transition distributions but the normal distribution delivers a closed form solution.
} 
in a lower expected value relative to the undistorted expected value. Second, a higher variance baseline continuation value, or equivalently a higher variance baseline transition measure, results in a more distorted expected value for some given $\theta$. A higher variance baseline continuation value means that the policymaker is putting more weight on potential extreme outcomes. This frees up the evil agent to select larger distortions since this additional weight on extreme values in the baseline model will tend to reduce Kullback-Leibler divergence.

Figure 4 shows that as $\theta \rightarrow \infty$, the distorted expected value approaches its undistorted value and we approach a subjective expected utility setting. As $\theta$ decreases, the distorted expected continuation value declines. Smaller values of $\theta$ make her expected future look worse and induces her to select policies that better guard against misspecifications that would reduce her future welfare. There typically exists some point $\bar{\theta}$, where the problem "breaks down" for $\theta<\bar{\theta} .^{24}$ I calibrate $\theta$ by selecting close to the breakdown point that would induce high levels of concern for model misspecification. ${ }^{25}$ For both frameworks I set $\theta=3.9$. In Section III.B I show how varying the concern for model misspecification alters policy.

\section{The Policy Effects of Uncertainty and Con- cern for Model Misspecification}

The time $t$ optimal carbon tax is the shadow cost of time $t$ emissions, $e_{t}$, and is brought into dollar terms using the optimized time $t$ marginal utility of consumption. Time $t$ emissions affect the following time $t+1$ states: cumula-

\footnotetext{
${ }^{24}$ The problem breaks down because when $\theta<\bar{\theta}$, the exponential term in $T^{1}\left(\beta V_{t+1}\left(\boldsymbol{S}_{\boldsymbol{t}+\mathbf{1}}\right) \mid \theta\right)$ is large (e.g. it is much larger than $\left.10^{100}\right)$ so that it is equivalent to numerical infinity. The problem is then no longer well-defined.

${ }^{25}$ Hansen and Sargent (2008) demonstrate how to calibrate $\theta$ using detection error probabilities in a linear control setting. Athanassoglou and Xepapadeas (2012) formally derive a closed form solution for the worst case misspecification exploiting a linear-quadratic model. Since the DICE model is highly non-linear I take an approach similar to Gonzalez (2007) where I explore how policy changes under different values of the penalty parameter in Section III.B.
} 
tive emissions (and thus temperature), the fraction of output remaining after damages $\left(\mathcal{L}_{t+1}=\frac{1}{1+d_{1}\left[T_{t+1}^{s}\right]^{d_{2}} \omega_{t+1}}\right)$, and the location and scale parameters. ${ }^{26}$ From here on I call $\mathcal{L}_{t+1}$ fractional net output. ${ }^{27}$ Without loss of generality, consider the optimal carbon tax for a policymaker using robust control. The shadow cost of emissions is the negative partial derivative of the right hand side of equation (8) with respect to time $t$ emissions, $e_{t}$

$$
\operatorname{Tax}_{t}=\frac{\beta_{t}}{u^{\prime}\left(c_{t}\right)} \mathbb{E}_{d_{2}}\left[\frac{\mathbb{E}_{d_{1}, \omega_{t+1}}\left[\exp \left(\frac{-\beta_{t} V_{t+1}}{\theta}\right)\left(\frac{-\partial V_{t+1}}{\partial \mathbf{S}_{t+1}} \frac{\partial \mathbf{S}_{t+1}}{\partial e_{t}}\right)\right]}{\mathbb{E}_{d_{1}, \omega_{t+1}}\left[\exp \left(\frac{-\beta_{t} V_{t+1}}{\theta}\right)\right]}\right]
$$

where the expectations are separated because of the risk sensitivity operator $T_{1}$. The application of robust control introduces a new term, $\exp \left(\frac{-\beta_{t} V_{t+1}}{\theta}\right)$, which when normalized by its expectation, corresponds to the twisting factor of the worst-case transition distortion induced by the risk sensitivity operator (Hansen and Sargent, 2007).

Now I will focus on only the key steps and terms in the derivation in order to convey the intuition for the different carbon tax channels as cleanly as possible. The full carbon tax derivation can be found in the online appendix. Passing the expectations through and exploiting covariance identities, the carbon tax equation can be rearranged to recover a conventional carbon tax expression (first three lines) and an additively separable adjustment for robust control (fourth line)

$$
\begin{aligned}
\operatorname{Tax}_{t} & =\frac{\beta_{t}}{u^{\prime}\left(c_{t}\right)}\left\{\mathbb{E}_{d_{2}, d_{1}, \omega_{t+1}}\left[\frac{-\partial V_{t+1}}{\partial E_{t+1}}\right] \frac{\partial E_{t+1}}{\partial e_{t}}\right. \\
& +\mathbb{E}_{d_{2}, d_{1}, \omega_{t+1}}\left[\frac{-\partial V_{t+1}}{\partial \mathcal{L}_{t+1}}\right] E_{d_{2}, d_{1}, \omega_{t+1}}\left[\frac{\partial \mathcal{L}_{t+1}}{\partial e_{t}}\right]+\operatorname{cov}_{d_{2}, d_{1}, \omega_{t+1}}\left(\frac{-\partial V_{t+1}}{\partial \mathcal{L}_{t+1}}, \frac{\partial \mathcal{L}_{t+1}}{\partial e_{t}}\right) \\
& +\operatorname{cov}_{d_{2}, d_{1}, \omega_{t+1}}\left(\frac{-\partial V_{t+1}}{\partial \mu_{t+1}^{e}}, \frac{\partial \mu_{t+1}^{e}}{\partial e_{t}}\right)+\mathbb{E}_{d_{2}, d_{1}, \omega_{t+1}}\left[\frac{-\partial V_{t+1}}{\partial \Sigma_{t+1}^{e}}\right] \frac{\partial \Sigma_{t+1}^{e}}{\partial e_{t}}
\end{aligned}
$$

\footnotetext{
${ }^{26}$ In expectation, the effect of time $t$ emissions on the time $t+1$ location parameter is zero but we will see that a covariance term arises that may not be zero.

${ }^{27}$ I use fractional net output as a state instead of damages to obtain a bounded domain, $\mathcal{L}_{t+1} \in[0,1]$.
} 


$$
\left.+\mathbb{E}_{d_{2}}\left[\operatorname{cov}_{d_{1}, \omega_{t+1}}\left(\frac{-\partial V_{t+1}}{\partial \mathbf{S}_{\mathbf{t}+\mathbf{1}}} \frac{\partial \mathbf{S}_{\mathbf{t}+\mathbf{1}}}{\partial e_{t}}, \frac{\exp \left(\frac{-\beta_{t} V_{t+1}}{\theta}\right)}{\mathbb{E}_{d_{1}, \omega_{t+1}}\left[\exp \left(\frac{-\beta_{t} V_{t+1}}{\theta}\right)\right]}\right)\right]\right\}
$$

Next, I introduce two state vectors. The first is the expected time $t+1$ state vector from the perspective of the time $t$ information set

$$
v:=\left\{k_{t+1}, E_{t+1}, \mathbb{E}_{d_{2}, d_{1}, \omega_{t+1}}\left[\mathcal{L}_{t+1}\right], \mu_{t}^{e}, \Sigma_{t+1}^{e}\right\}
$$

which recognizes that $\mathbb{E}_{d_{2}, d_{1}, \omega_{t+1}}\left[\mu_{t+1}^{e}\right]=\mu_{t}^{e}$ given time $t$ beliefs, and the other states without expectations transition deterministically. ${ }^{28}$ The second is the certainty state

$$
c e:=\left\{k_{t+1}, E_{t+1},\left.\mathcal{L}_{t+1}\right|_{d_{1}=\exp \left(\mu_{c}+\frac{1}{2} \sigma_{c}^{2}\right), \omega_{t+1}=\exp \left(\mu_{\omega}+\frac{1}{2} \sigma_{\omega}^{2}\right)}, \mu_{t}^{e}, 0\right\},
$$

which is the time $t+1$ state that would be reached if all random variables were set equal to their means and the prior variance was set to zero.

Last, I decompose the tax into channels that map closely into the consumptionsaving literature with a two step procedure. In the first step of the decomposition I perform a second-order Taylor expansion of the expectations over the value function partial derivative terms on the first three lines of equation (9) around the expected state $v{ }^{29}$ In the second step I add and subtract two sets of identical terms evaluated at the certainty state ce to the Taylor expansion. This results in an expression that I will group into seven different channels. ${ }^{30}$

To economize on space I omit both the leading $\beta_{t} / u^{\prime}\left(c_{t}\right)$ term in equation (9) and the Taylor expansion terms for the value function partial deriva-

\footnotetext{
${ }^{28} \mu_{t+1}^{e}$ has a normal distribution and $\mathcal{L}_{t+1}$ does not have a named distribution.

${ }^{29}$ I require the value function to be three times differentiable in a number of the arguments. This is not necessarily guaranteed by having the utility function be three times differentiable and proving that my analytic value function is three times differentiable is not trivial. Here I am using high order polynomial approximations to the value function that are greater than third order so my approximating value function is trivially three times differentiable.

${ }^{30}$ Lemoine and Rudik (2017) similarly demonstrate how to use Taylor expansions of the value function to gain insight into how uncertainty over the climate's sensitivity to emissions drives optimal policy.
} 
tives with respect to the scale parameter $\Sigma_{t+1}^{e}$ since they will be the same as the other states. The derivation of the full Taylor expansions are in the online appendix. For the subsequent analysis, recognize that $\partial E_{t} / \partial e_{t}=1$, and that fractional net output is decreasing in emissions $\left(\partial \mathcal{L}_{t+1} / \partial e_{t}<0\right)$.

\section{Channel 1: Certainty tax}

$$
\left.\frac{-\partial V_{t+1}}{\partial E_{t+1}}\right|_{c e}+\left.\left.\frac{-\partial V_{t+1}}{\partial \mathcal{L}_{t+1}}\right|_{c e} \frac{\partial \mathcal{L}_{t+1}}{\partial e_{t}}\right|_{c e}
$$

The first channel is the certainty tax. The certainty tax arises from adding $\left.\frac{-\partial V_{t+1}}{\partial E_{t+1}}\right|_{c e}+\left.\left.\frac{-\partial V_{t+1}}{\partial \mathcal{L}_{t+1}}\right|_{c e} \frac{\partial \mathcal{L}_{t+1}}{\partial e_{t}}\right|_{c e}$ to the full Taylor expansion around the expected state $v$. The certainty tax is the shadow cost of an additional unit of emissions when all uncertain terms are perfectly known and fixed at their time $t$ means. This is the tax that would be set by a policymaker that happened to be at the current time $t$ state $\mathbf{S}_{t}$ and states transitioned deterministically (Lemoine and Rudik, 2017).

\section{Channel 2: State uncertainty adjustment}

$$
\left(\left.\frac{-\partial V_{t+1}}{\partial E_{t+1}}\right|_{\zeta}+\left.\frac{-\partial V_{t+1}}{\partial \mathcal{L}_{t+1}}\right|_{\zeta} \mathbb{E}_{d_{2}, d_{1}, \omega_{t+1}}\left[\frac{\partial \mathcal{L}_{t+1}}{\partial e_{t}}\right]\right)-\left(\left.\frac{-\partial V_{t+1}}{\partial E_{t+1}}\right|_{c e}+\left.\left.\frac{-\partial V_{t+1}}{\partial \mathcal{L}_{t+1}}\right|_{c e} \frac{\partial \mathcal{L}_{t+1}}{\partial e_{t}}\right|_{c e}\right)
$$

The second channel is the state uncertainty adjustment. This channel alters the certainty tax so that it correctly accounts for how uncertainty affects the expected time $t+1$ state. It is the difference in the tax evaluated at the expected state and the tax evaluated at the certainty state; the second term arises from subtracting the certainty tax terms from the full Taylor expansion. The rest of the channels account for how uncertainty affects the expected shadow cost of emissions because of curvature in the value function partial derivatives. 


\section{Channel 3: Precautionary abatement}

$$
\begin{aligned}
& \left.\frac{1}{2} \frac{-\partial^{3} V_{t+1}}{\partial E_{t+1} \mathcal{L}_{t+1}^{2}}\right|_{\zeta} \operatorname{var}_{d_{2}, d_{1}, \omega_{t+1}}\left(\mathcal{L}_{t+1}\right)+\left.\frac{1}{2} \frac{-\partial^{3} V_{t+1}}{\partial \mathcal{L}_{t+1}^{3}}\right|_{\zeta} \mathbb{E}_{d_{2}, d_{1}, \omega_{t+1}}\left[\frac{\partial \mathcal{L}_{t+1}}{\partial e_{t}}\right] \operatorname{var}_{d_{2}, d_{1}, \omega_{t+1}}\left(\mathcal{L}_{t+1}\right) \\
& +\left.\frac{-\partial^{3} V_{t+1}}{\partial E_{t+1} \partial \mathcal{L}_{t+1} \partial \mu_{t+1}^{e}}\right|_{\zeta} \operatorname{cov}_{d_{2}, d_{1}, \omega_{t+1}}\left(\mathcal{L}_{t+1}, \mu_{t+1}^{e}\right)+\left.\frac{-\partial^{3} V_{t+1}}{\partial \mathcal{L}_{t+1}^{2} \partial \mu_{t+1}^{e}}\right|_{\zeta} \mathbb{E}_{d_{2}, d_{1}, \omega_{t+1}}\left[\frac{\partial \mathcal{L}_{t+1}}{\partial e_{t}}\right] \operatorname{cov}_{d_{2}, d_{1}, \omega_{t+1}}\left(\mathcal{L}_{t+1}, \mu\right.
\end{aligned}
$$

The third channel is the precautionary abatement motive. The numerical results indicate that this channel tends to increase the carbon tax when not learning about $d_{2}$ and decrease the carbon tax when learning about $d_{2}$. The third derivative of utility corresponds to how uncertainty about future consumption affects the policymaker's contemporaneous saving decision (Leland, 1968; Dreze and Modigliani, 1972; Kimball, 1990). If uncertainty about future consumption increases contemporaneous saving, the agent is said to exhibit prudence. In a climate-economy setting, abatement is a form of environmental saving: increasing current abatement means that the policymaker forgoes a sure consumption payoff now for increased consumption later due to lower future damages. The second term on the first line captures this most clearly. When not learning, the third derivative of her continuation value with respect to fractional net output is positive so that

$$
\left.\frac{1}{2} \frac{-\partial^{3} V_{t+1}}{\partial \mathcal{L}_{t+1}^{3}}\right|_{\zeta} \mathbb{E}_{d_{2}, d_{1}, \omega_{t+1}}\left[\frac{\partial \mathcal{L}_{t+1}}{\partial e_{t}}\right] \operatorname{var}_{d_{2}, d_{1}, \omega_{t+1}}\left(\mathcal{L}_{t+1}\right)>0,
$$

and the policymaker increases abatement and her optimal carbon tax. The opposite is true when learning. The precautionary motive also scales in size with the variance of future output.

The first term on the first line captures similar precautionary abatement motives in the face of uncertain output. If this term increases abatement then the agent would be called cross-prudent (Gollier, 2010). The policymaker is cross-prudent if she would prefer to have a mean-zero risk attached to fractional net output when cumulative emissions are lower rather than when they are higher.

The second line captures precautionary abatement motives because of cross- 
prudence, and these channels generally increase the optimal carbon tax. Here, the policymaker abates more because of co-variability between fractional net output and her beliefs about the damage exponent. In the numerical results the covariance terms are generally negative since smaller than expected $\mathcal{L}_{t+1}$ is the signal that the policymaker would need to receive to revise her expectations about $d_{2}$ upward. This covariance matters because when future beliefs about damages are uncertain, then future output and future consumption appear to be even more variable. Whatever information the policymaker receives about output in the future feeds back onto her payoffs through her expectations.

\section{Channel 4: Signal smoothing}

$\left.\frac{1}{2} \frac{-\partial^{3} V_{t+1}}{\partial E_{t+1} \partial \mu_{t+1}^{e}{ }^{2}}\right|_{\zeta} \operatorname{var}_{d_{2}, d_{1}, \omega_{t+1}}\left(\mu_{t+1}^{e}\right)+\left.\frac{1}{2} \frac{-\partial^{3} V_{t+1}}{\partial \mathcal{L}_{t+1} \partial \mu_{t+1}^{e}{ }^{2}}\right|_{\zeta} \mathbb{E}_{d_{2}, d_{1}, \omega_{t+1}}\left[\frac{\partial \mathcal{L}_{t+1}}{\partial e_{t}}\right] \operatorname{var}_{d_{2}, d_{1}, \omega_{t+1}}\left(\mu_{t+1}^{e}\right)$

The fourth channel is the signal smoothing motive which tends to increase the optimal carbon tax in the numerical results. This channel captures one effect of learning on the optimal tax. The second derivative of the value function with respect to the location parameter, $-\partial^{2} V_{t+1} / \partial \mu_{t+1}^{e}{ }^{2}$, captures how well the policymaker can use new information to smooth welfare over possible values of $d_{2}$. Since a higher location parameter for the policymaker's beliefs strictly increases her expectation of $d_{2}$, and since $-\partial^{2} V_{t+1} / \partial \mu_{t+1}^{e}{ }^{2}>0$ in the simulations, the marginal welfare cost of a higher $d_{2}$ belief is increasing and convex. If there is not much curvature in her beliefs and $\partial^{2} V_{t+1} / \partial \mu_{t+1}^{e}{ }^{2}$ is small, then she is able to smooth welfare effectively, but the larger $\partial^{2} V_{t+1} / \partial \mu_{t+1}^{e}{ }^{2}$ is in magnitude, the less she can smooth welfare, and the more costly a bad signal of $d_{2}$ becomes.

The triple derivatives then indicate how a marginal increase in cumulative emissions or fractional net output affects the policymaker's ability to smooth welfare in response to new information. Indeed, additional emissions decrease her ability to smooth welfare $\left(-\partial^{3} V_{t+1} / \partial E_{t+1} \partial \mu_{t+1}^{e}{ }^{2}>0\right)$, and having a greater fraction of her gross output would increase her ability to smooth welfare $\left(-\partial^{3} V_{t+1} / \partial \mathcal{L}_{t+1} \partial \mu_{t+1}^{e}{ }^{2}<0\right)$. Additional emissions results in greater 
damages and less output to be able to use towards abatement, while additional fractional net output has the opposite effect. In these cases, additional variability in future beliefs magnifies this effect and increases the optimal tax.

\section{Channel 5: Output insurance}

$$
\operatorname{cov}_{d_{2}, d_{1}, \omega_{t+1}}\left(\frac{-\partial V_{t+1}}{\partial \mathcal{L}_{t+1}}, \frac{\partial \mathcal{L}_{t+1}}{\partial e_{t}}\right)
$$

The fifth channel is the output insurance channel. In simulations, this channel decreases the optimal carbon tax over the first 90 years when not learning before eventually increasing the carbon tax. It always increases the optimal carbon tax in the simulations with learning. Output insurance increases the optimal carbon tax if and only if the covariance is positive. The first term in the covariance captures the marginal welfare cost of reducing fractional net output, and the second term in the covariance captures the marginal reduction in fractional net output from emissions. The policymaker cares about the covariance of returns to emissions reductions with marginal utility. This channel increases the optimal carbon tax if emissions reductions are most effective in preserving output when output is most valuable to welfare.

\section{Channel 6: Learning insurance}

$$
\operatorname{cov}_{d_{2}, d_{1}, \omega_{t+1}}\left(\frac{-\partial V_{t+1}}{\partial \mu_{t+1}^{e}}, \frac{\partial \mu_{t+1}^{e}}{\partial e_{t}}\right)
$$

The sixth channel is the learning insurance channel. This channel generally increases the optimal carbon tax in the simulations. Learning insurance motives are similar to output insurance motives in that the policymaker cares about the covariance between the effect of emissions reductions on expectations and marginal welfare. The covariance term is generally positive so this channel reduces the optimal level of emissions. 


\section{Channel 7: Misspecification insurance}

$$
\mathbb{E}_{d_{2}}\left[\operatorname{cov}_{d_{1}, \omega_{t+1}}\left(\frac{-\partial V_{t+1}}{\partial \mathbf{S}_{\mathbf{t}+\mathbf{1}}} \frac{\partial \mathbf{S}_{\mathbf{t}+\mathbf{1}}}{\partial e_{t}}, \frac{\exp \left(\frac{-\beta_{t} V_{t+1}}{\theta}\right)}{\mathbb{E}_{d_{1}, \omega_{t+1}}\left[\exp \left(\frac{-\beta_{t} V_{t+1}}{\theta}\right)\right]}\right)\right]
$$

The final channel is the misspecification insurance channel. The magnitude and direction of the misspecification insurance channel depends on how the marginal welfare cost of emissions covaries with the worst-case distortion to the transition density. The misspecification insurance channel increases the carbon tax if and only if the covariance is positive. This channel increases the optimal carbon tax when not learning and slightly decreases the optimal carbon tax when learning. A decrease in the optimal carbon tax is consistent with previous findings that concerns about model misspecification increase motives for policy experimentation (Cogley et al., 2008).

\section{Results}

First, I show the mean optimal carbon tax trajectories and the corresponding climate outcomes over the next century for each of the four frameworks. Next, I vary the robust control penalty parameter to examine how the policymaker's concern for model misspecification affects policy. Then, I decompose the carbon taxes into each channel outlined in Section II to determine how uncertainty matters for policy. Finally, I analyze welfare outcomes under the different frameworks. In each simulation run, I randomly sample a vector of annual damage shocks $\left\{\omega_{2005}, \ldots, \omega_{2105}\right\}$ from the damage shock distribution. In cases where I take ex ante expectations of the optimal carbon tax, I also randomly sample a $\left\{d_{1}, d_{2}\right\}$ pair from their year 2005 prior distributions in each simulation. 


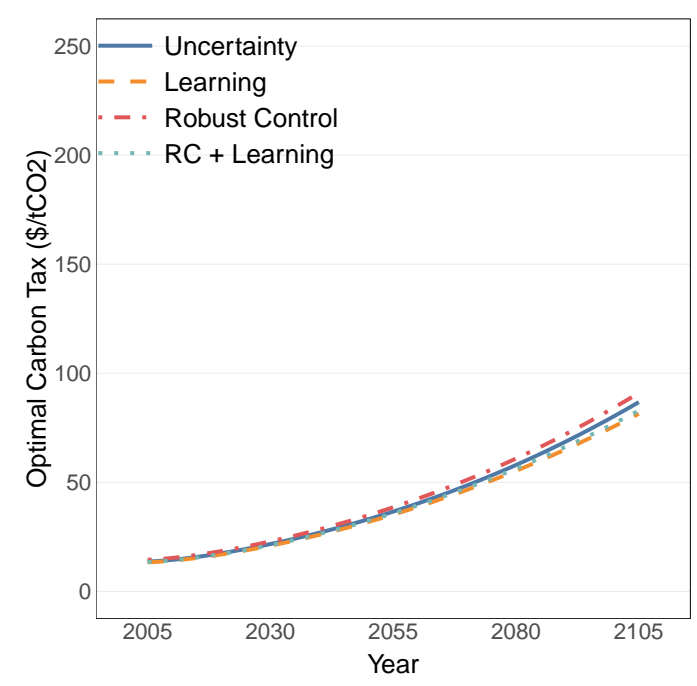

(a) Ex ante Expected Tax

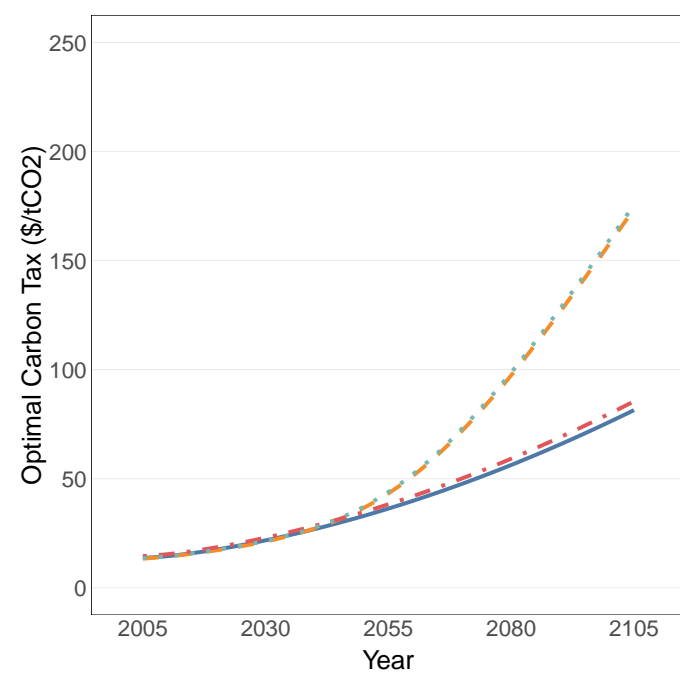

(c) $d_{1}=0.0056, d_{2}=3.0$

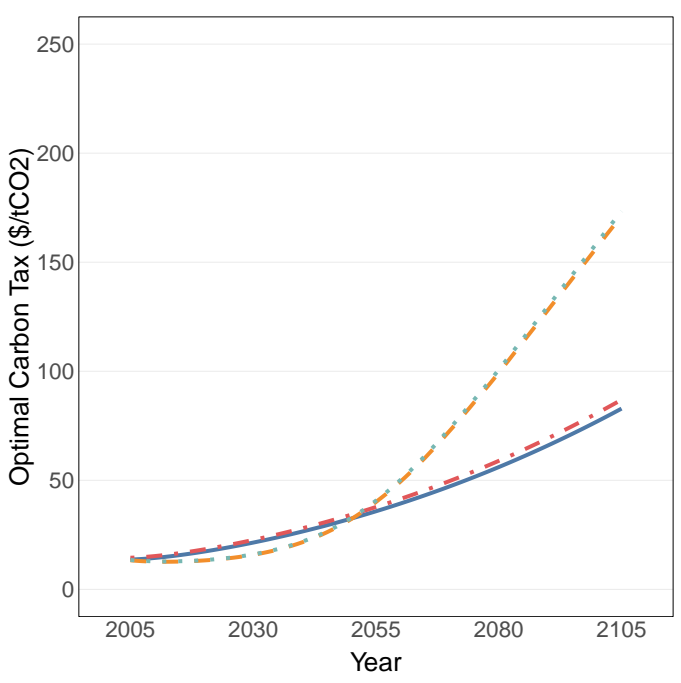

(b) $d_{1}=0.01, d_{2}=1.88$

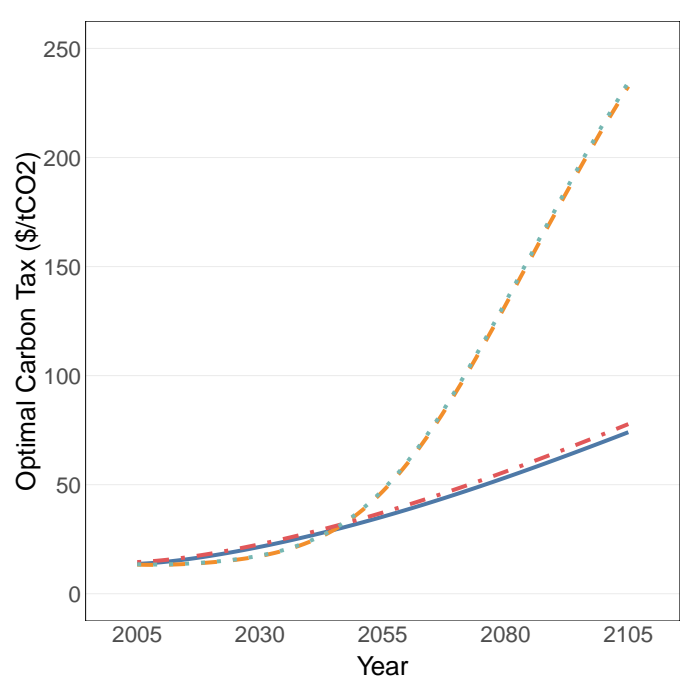

(d) $d_{1}=0.01, d_{2}=3.0$

Figure 5: The ex ante expected optimal carbon tax over 50,000 simulations for each framework (top left), and the expected optimal carbon tax under specific damage functions over 5,000 simulations for each framework (other three panels). For the top left panel, each of the 50,000 simulations randomly samples a different set of damage parameters and damage shocks $\left\{d_{1}, d_{2}, \omega_{2005}, \ldots, \omega_{2105}\right\}$. For the other three panels, all simulations use the same damage parameters listed below the panel, and a random sample of damage shocks $\left\{\omega_{2005}, \ldots, \omega_{2105}\right\}$. 


\section{III.A Optimal Carbon Tax Trajectories}

Figure 5 displays the first century's optimal carbon tax trajectories in $\$ / \mathrm{tCO}_{2} \cdot{ }^{31}$ The top left panel displays the ex ante expected carbon tax trajectories averaged over 50,000 simulations. Each simulation randomly draws a pair of damage function parameters and a sequence of annual damage shocks $\left\{d_{1}, d_{2}, \omega_{2005}, \ldots, \omega_{2105}\right\}$ from their distributions defined by the estimated parameters in Table 1 . The remaining three panels display results averaged over 5,000 simulations when $d_{1}$ and $d_{2}$ are fixed to a single value across all 5,000 simulations, but each simulation still randomly draws a sequence of annual damage shocks $\left\{\omega_{2005}, \ldots, \omega_{2105}\right\}$. The top right panel sets the true coefficient to 0.01 and the true exponent equal to its expectation, the bottom left panel sets the true coefficient to its expectation and the true exponent to 3.0, and the bottom right panel sets the true coefficient to 0.01 and the true exponent to 3.0.

The top left panel plots the ex ante expected carbon tax trajectories, i.e. the tax trajectories the policymaker expects to set over the first 100 years given her 2005 information set. Under the uncertainty framework, the policymaker begins her optimal tax in 2005 at $\$ 13.68 / \mathrm{tCO}_{2}$ and expects to ramp it up to $\$ 86.76 / \mathrm{tCO}_{2}$ in 2105 . When the policymaker is able to learn $d_{2}$ over time, she sets an initial carbon tax 2 percent lower at $\$ 13.34 / \mathrm{tCO}_{2}$, which on average rises to $\$ 81.44 / \mathrm{tCO}_{2}$ at the end of the century. Applying robust control to guard against potential model misspecification tends to increase the tax when not updating beliefs. In 2005 the robust control tax begins slightly higher than the uncertainty framework at $\$ 14.52 / \mathrm{tCO}_{2}$. Over the first century, the robust control tax is expected to increase faster than the uncertainty tax and reaches $\$ 90.99 / \mathrm{tCO}_{2}$ at the end of the century. Applying robust control on top of learning increases the initial carbon tax with learning by $\$ 0.15 / \mathrm{tCO}_{2}$, and by 2105 the ex ante expected carbon tax with both robust control and learning is $\$ 1.41 / \mathrm{tCO}_{2}$ higher than just learning alone.

The top right panel shows the mean carbon tax trajectories when the damage coefficient is about double the policymaker's expectation and the damage

\footnotetext{
${ }^{31}$ Some papers report carbon taxes in terms of dollars per ton of carbon. The unit conversion is 11 tons of $\mathrm{CO}_{2}$ to three tons of carbon.
} 
exponent is equal to her expectation. Initial carbon taxes start at the same level as in the top left panel since the policymaker is making decisions with the same information set. When facing a higher damage coefficient, the carbon taxes under the uncertainty and robust control frameworks ramp up at a slower rate, reaching $\$ 82.89 / \mathrm{tCO}_{2}$ for the uncertainty framework and $\$ 86.95 / \mathrm{tCO}_{2}$ for the robust control framework. The large damage coefficient results in more damage and less output to allocate toward abatement so the policymaker sets a lower carbon tax. The greater level of damages acts like a negative income effect. The two frameworks with learning have carbon tax trajectories that initially decrease. Temperature starts below $1^{\circ} \mathrm{C}$ and the damage function has a monomial form, so when a learning policymaker observes higher damages in the early years she attributes it to a smaller damage exponent and revises her expectation about $d_{2}$ downward to approximately 1.6. When temperature crosses the $1^{\circ} \mathrm{C}$ threshold, she starts revising her beliefs about $d_{2}$ upward and begins quickly increasing her carbon tax which reaches $\$ 171.34 / \mathrm{tCO}_{2}$ and $\$ 173.37 / \mathrm{tCO}_{2}$ in 2105 under the learning and robust control and learning frameworks. ${ }^{32}$

The bottom left panel shows the mean carbon tax trajectories when the damage coefficient is equal to the policymaker's expectation and the damage exponent is about double her expectation. Similar to the top right panel, the non-learning frameworks' carbon taxes are lower than ex ante expectations because the policymaker has less output to allocate toward abatement under a more severe damage function. Optimal carbon taxes reach only $\$ 81.42 / \mathrm{tCO}_{2}$ and $\$ 85.44 / \mathrm{tCO}_{2}$ in 2105 for the uncertainty and robust control frameworks. Over the first few decades, carbon taxes under the learning and robust control and learning frameworks are similar to ex ante expected levels plotted in the top left panel. Damage functions with different exponents yield similar levels of damages at low temperatures so its difficult to statistically distinguish them. In this setting, the policymaker's mean belief under the learning and $\mathrm{RC}+\mathrm{L}$ frameworks only increases to about 1.9 by 2035 when temperature reaches about $1.1^{\circ} \mathrm{C}$. In the second half of the century the policymaker begins

\footnotetext{
${ }^{32}$ The policymaker expects $d_{2} \approx 2.8$ at the end of the first century.
} 
Table 2: The ex ante expected optimal carbon tax in 2055 and $2105, \mathrm{CO}_{2}$ and temperature in 2105 , and the $5^{\text {th }}$ and $95^{\text {th }}$ percentiles (in parentheses) for each framework over 50,000 simulations. Each of the 50,000 simulations randomly samples a different set of damage parameters and damage shocks $\left\{d_{1}, d_{2}, \omega_{2005}, \ldots, \omega_{2105}\right\}$.

\begin{tabular}{lcccc}
\hline & \multicolumn{4}{c}{ Framework } \\
\cline { 2 - 5 } & Uncertainty & Learning & $\mathrm{RC}$ & $\mathrm{RC}+\mathrm{L}$ \\
\hline $2055 \operatorname{Tax}\left(\$ / \mathrm{tCO}_{2}\right)$ & 37 & 35 & 39 & 36 \\
& $(36,37)$ & $(25,46)$ & $(38,39)$ & $(25,47)$ \\
$2105 \operatorname{Tax}\left(\$ / \mathrm{tCO}_{2}\right)$ & 87 & 81 & 91 & 83 \\
& $(82,90)$ & $(30,138)$ & $(86,94)$ & $(30,140)$ \\
$2105 \mathrm{CO}_{2}($ parts per million $)$ & 613 & 620 & 611 & 618 \\
& $(612,613)$ & $(575,666)$ & $(610,611)$ & $(573,665)$ \\
$\left.2105 \mathrm{Temperature}^{\circ}{ }^{\circ} \mathrm{C}\right)$ & 2.08 & 2.10 & 2.07 & 2.10 \\
& $(2.08,2.08)$ & $(1.95,2.26)$ & $(2.07,2.07)$ & $(1.94,2.25)$ \\
\hline
\end{tabular}

learning more rapidly and quickly increases her carbon tax to $\$ 173.62 / \mathrm{tCO}_{2}$ and $\$ 175.41 / \mathrm{tCO}_{2}$ in 2105 for the learning and $\mathrm{RC}+\mathrm{L}$ frameworks.

The bottom right panel plots mean carbon tax trajectories when the damage coefficient and damage exponent are both higher than the policymaker's $e x$ ante expectation. This is the most severe damage function so the non-learning frameworks face their smallest output budgets and set carbon taxes of only $\$ 74.04 / \mathrm{tCO}_{2}$ and $\$ 77.77 / \mathrm{tCO}_{2}$ in 2105 . High damages generate high learning carbon taxes. In 2105 they reach $\$ 232.58 / \mathrm{tCO}_{2}$ for the learning framework and $\$ 234.47 / \mathrm{tCO}_{2}$ for the $\mathrm{RC}+\mathrm{L}$ framework. The learning policymakers attribute the high observed amounts of damages entirely to a large exponent, so that expectations about $d_{2}$ overshoot its true value and reach about 3.6 in 2105.

Table 2 displays the mean, $5^{\text {th }}$ and $95^{\text {th }}$ percentile outcomes for the 2055 carbon tax, the 2105 carbon tax, and 2105 atmospheric $\mathrm{CO}_{2}$ concentrations and and temperature. Learning results in significant variability in realized carbon taxes since the policymaker adapts her policy to the noisy information she receives about each simulation's specific $d_{2}$. After 50 years the learning 
carbon tax is about $\$ 2 / \mathrm{tCO}_{2}$ less than the uncertainty tax on average, but in some cases it may be nearly 30 percent lower or 30 percent higher. After 100 years the learning carbon tax is about 7 percent smaller than the uncertainty carbon tax on average, but it still displays substantial variability depending on the realized values of the damage function parameters and the damage shocks. After 50 years the mean robust control carbon tax is $\$ 2 / \mathrm{tCO}_{2}$ higher than the mean uncertainty carbon tax, but after 100 years the difference grows to $\$ 4 / \mathrm{tCO}_{2}$.

When learning, the policymaker allows $\mathrm{CO}_{2}$ to be 7 parts per million (ppm) higher in 2105, which results in greater warming compared to the uncertainty framework. Conversely, the more aggressive carbon tax in the robust control framework tends to keep $\mathrm{CO}_{2}$ lower than the uncertainty framework by 2 ppm in 2105. There is also significant variability in climatic outcomes when learning. The 90 percent confidence interval for $2105 \mathrm{CO}_{2}$ is about $90 \mathrm{ppm}$ wide. This is approximately the same as the real world change in atmospheric $\mathrm{CO}_{2}$ concentrations from 1960-2018.

\section{III.B The Effect of Concern for Model Misspecification}

Figure 6 shows how changing the level of the policymaker's concern about model misspecification affects the initial optimal carbon tax. Smaller values of the penalty parameter indicate greater misspecification concern. The left panel of Figure 6 displays the optimal carbon tax as a function of the penalty parameter for the robust control framework. When the penalty parameter is sufficiently large, the year 2005 optimal carbon tax is effectively equal to the optimal carbon tax of the uncertainty framework. As the penalty parameter declines and we move to the left on the plot, the optimal carbon tax increases, and on this plot, peaks at $\$ 14.52 / \mathrm{tCO}_{2}$ when the penalty parameter is 3.9. Decreasing it further results in the problem breaking down so the model no longer solves.

The right panel of Figure 6 displays the initial optimal carbon tax for the robust control and learning framework. Again, a sufficiently large penalty 


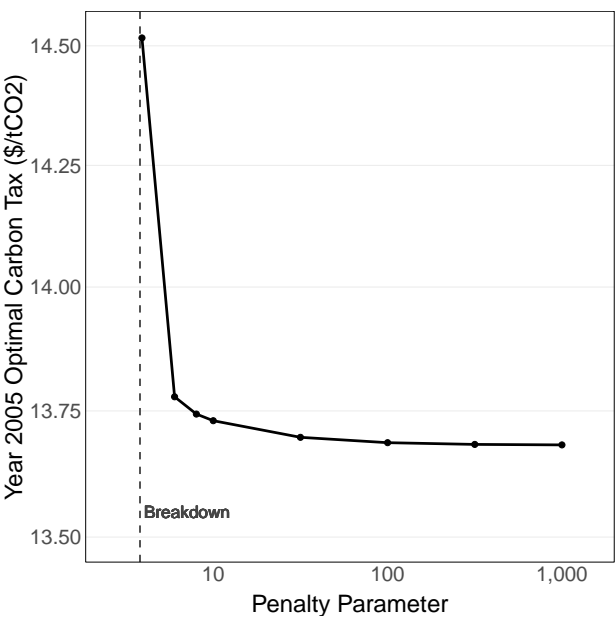

(a) Robust Control Framework

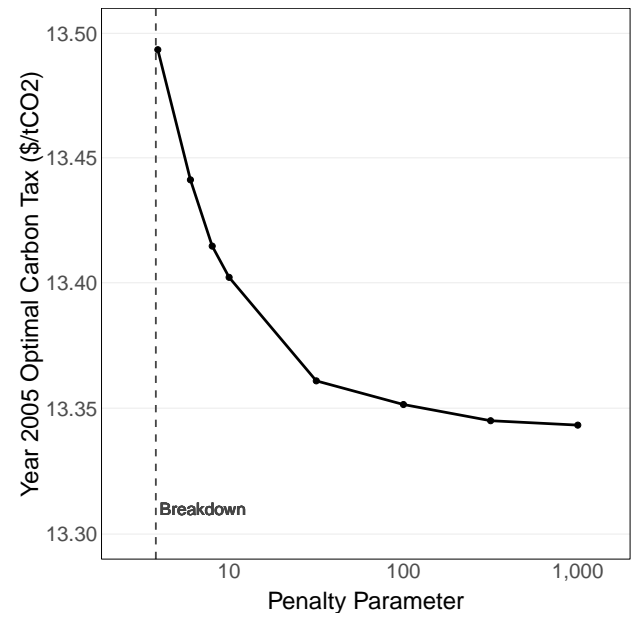

(b) $\mathrm{RC}+\mathrm{L}$ Framework

Figure 6: The year 2005 optimal carbon taxes as a function of $\theta$ for the robust control framework (left) and the $\mathrm{RC}+\mathrm{L}$ framework (right). The dashed line indicates the approximate location of $\bar{\theta}$ where the problem breaks down. Note that each panel has a different $\mathrm{y}$-axis scale and both $\mathrm{x}$ axes are on a log10 scale.

parameter leads the optimal carbon tax to be effectively equal to the learning framework without robust control. Decreasing the penalty parameter to 3.9 , right before the problem breaks down, increases the 2005 carbon tax to $\$ 13.49 / \mathrm{tCO}_{2}$.

\section{III.C Decomposing the Optimal Carbon Tax}

Figure 7 plots the six channels that capture uncertainty's effect on the optimal carbon taxes plotted in the top left panel of Figure $5 .^{33}$ Note that all panels have different scales to better display differences between the different frameworks. The certainty tax is in the appendix. Panel (a) plots the state uncertainty adjustment, the strongest of the channels. For the uncertainty framework and robust control framework, the state uncertainty adjustment

\footnotetext{
${ }^{33}$ The total effect of these channels is to increase the optimal carbon tax above the certainty tax by up to 1 percent for the uncertainty framework, 5 percent for the robust control framework, 5 percent for the learning framework, and 7 percent for the $\mathrm{RC}+\mathrm{L}$ framework.
} 


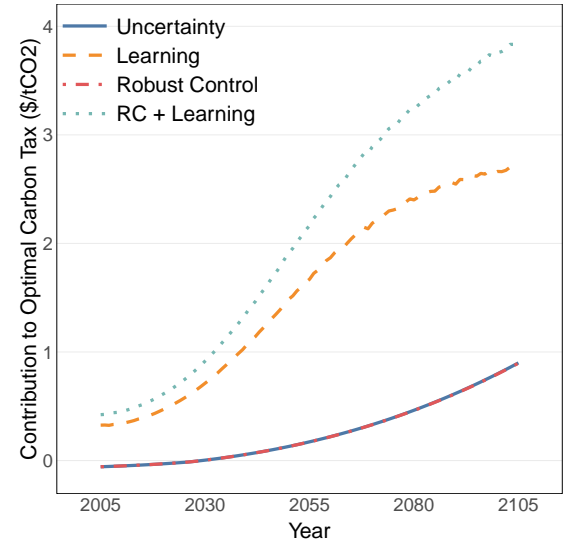

(a) State Uncertainty Adjustment

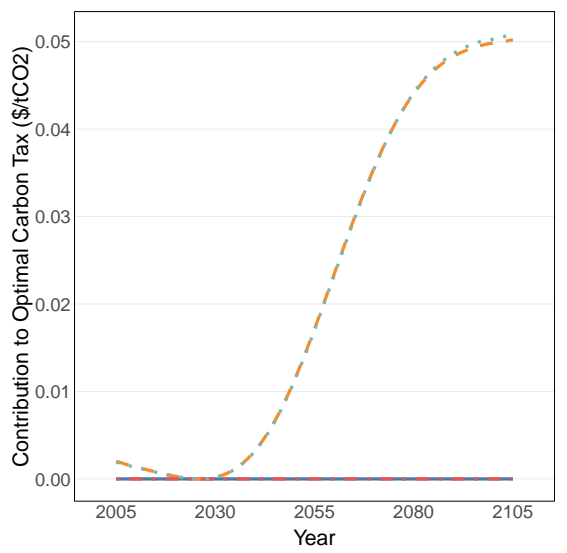

(c) Signal Smoothing

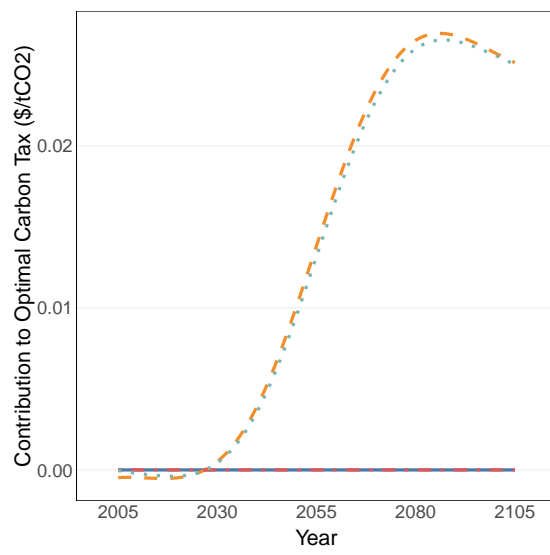

(e) Learning Insurance

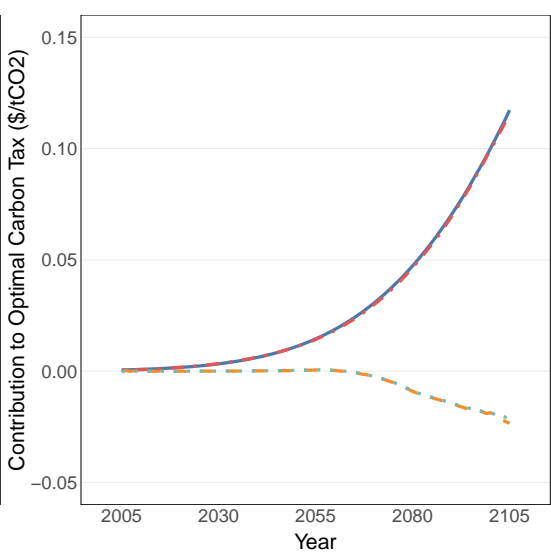

(b) Precautionary Abatement

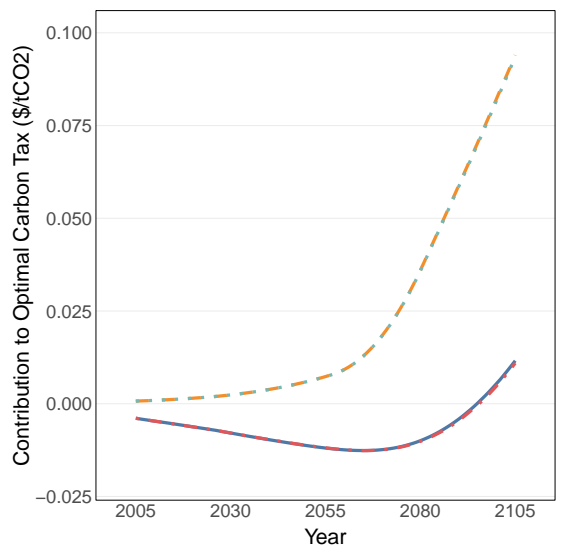

(d) Output Insurance

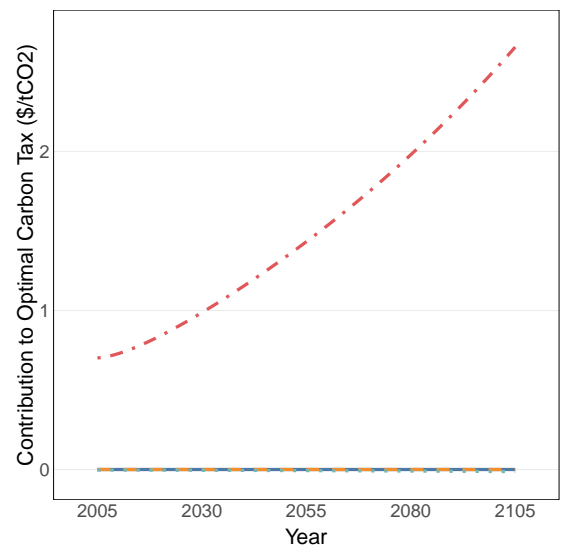

(f) Misspecification Insurance

Figure 7: The ex ante expectations of the six carbon tax channels related to uncertainty. The expectations are taken over 50,000 simulations, where each simulation randomly samples a different set of damage parameters and damage shocks $\left\{d_{1}, d_{2}, \omega_{2005}, \ldots, \omega_{2105}\right\}_{34}$ Note that each panel has a different $\mathrm{y}$-axis scale. 
begins slightly below zero but becomes positive at 2030 and then grows in magnitude over time to about $\$ 1 / \mathrm{tCO}_{2}$ by 2105 . The change in sign is driven by the curvature of fractional net output in the three random variables. At lower levels of warming, fractional net output is convex in $d_{1}$ and $\omega_{t+1}$, and concave in $d_{2}$. The convexity in $d_{1}$ and $\omega_{t+1}$ dominates early on and makes fractional net output larger at the expected state versus the certainty state. This results in a negative state uncertainty adjustment because on the margin, the shadow cost of emissions is smaller if output is higher. As temperature rises, the concavity in $d_{2}$ begins to dominate, making the uncertainty adjustment positive. For the two frameworks with learning, the state uncertainty adjustment is always positive, larger in magnitude than without learning, and monotonically increases in size from $\$ 0.33 / \mathrm{tCO}_{2}$ and $\$ 0.42 / \mathrm{tCO}_{2}$ in 2005 to $\$ 2.71 / \mathrm{tCO}_{2}$ and $\$ 3.86 / \mathrm{tCO}_{2}$ in 2105 . Learning makes future beliefs variable and thus future states are more variable. This tends to increase the optimal carbon tax.

Panel (b) plots the precautionary abatement motive. Initially, precautionary abatement is effectively zero since variability in damages is negligible when temperature is low. Damage variability increases as temperature rises, leading to more precautionary abatement for the non-learning frameworks because the third derivative of their value functions with respect to fractional net output is positive, and less precautionary abatement for the frameworks with learning because the third derivative of their value functions with respect to fractional net output is negative. In the learning frameworks, increased damage variability is partially offset by the policymaker resolving uncertainty so this channel's magnitude is dampened.

Panel (c) plots the signal smoothing channel, which is specific to the frameworks with learning. This channel increases the optimal carbon tax because emissions hinder the policymaker's ability to smooth welfare. The signal smoothing channel starts below $\$ 0.01 / \mathrm{tCO}_{2}$ in 2005 and declines as her future beliefs become less variable. As temperature rises, emissions increasingly reduce the policymaker's ability to smooth welfare to new information. This begins dominating the decrease in belief variability around 2030 and the signal 
smoothing channel increases thereafter to $\$ 0.05 / \mathrm{tCO}_{2}$ in 2105 .

Panel (d) displays the effect of output insurance. This channel eventually increases the optimal carbon tax for all frameworks. This channel is near zero at first since surface temperature is low and there is not much variability in the marginal effect of emissions on fractional net output. For the non-learning frameworks this channel decreases initially because the covariance between the effect of emissions on fractional net output with the marginal welfare cost of less fractional net output is negative, but it eventually becomes positive and reaches $\$ 0.01 / \mathrm{tCO}_{2}$. For the learning frameworks the size of the channel grows at an increasing rate to approximately $\$ 0.09 / \mathrm{tCO}_{2}$.

Panel (e) displays the effect of learning insurance. This channel tends to have the smallest impact out of all six channels. It generally increases the optimal carbon tax because additional emissions tend to increase future mean beliefs about $d_{2}\left(\partial \mu_{t+1}^{e} / \partial e_{t}>0\right)$ when welfare is most sensitive to increases in the mean belief about $d_{2}\left(-\partial V_{t+1} / \partial \mu_{t+1}^{e}>0\right)$. This channel is near zero at first since temperature is low and there is not much variability in the marginal effect of emissions on fractional net output. The size of the channel grows at an increasing rate to approximately $\$ 0.03 / \mathrm{tCO}_{2}$ at 2085 before declining.

Panel (f) plots the misspecification insurance channel. The channel increases the carbon tax when not learning and decreases the carbon tax when learning. In both cases, this channel begins small as there is less variability early on when temperature is low, but the size of misspecification insurance grows over time for both frameworks using robust control. This is because the marginal benefit of emissions reductions and the distortion to the transition density become larger and increasingly correlated. The size of misspecification insurance reaches over $\$ 2 / \mathrm{tCO}_{2}$ without learning, and about $-\$ 0.02 / \mathrm{tCO}_{2}$ when learning. A negative effect of robust control on a learning carbon tax is consistent with findings of increased experimentation motives in other settings (Cogley et al., 2008). A learning policymaker can accelerate the learning process by allowing faster warming. Robust control amplifies this experimentation motive. ${ }^{34}$

\footnotetext{
${ }^{34}$ The scale parameter is strictly decreasing in temperature so allowing temperature to
} 


\section{III.D Welfare Implications of Learning and Robust Con- trol}

Next I investigate the relative welfare performance of the frameworks. First, I quantify ex ante welfare differences assuming that the policymaker has correctly specified her model so that the true damage function parameters are drawn from the distributions in Section I.C. Results for the learning framework gives us the expected benefits of updating the distribution over $d_{2}$, while the results for the robust control framework gives us the costs of designing policy to guard against misspecifications when we have actually specified damages correctly. ${ }^{35}$

Second, I quantify ex post welfare when the policymaker's approximating damage model may be misspecified and the true damage function is instead one of three catastrophic damage functions. The first two are the damage functions of Weitzman (2012) and Dietz and Stern (2015) where damages follow close to DICE at low levels of warming, but rise rapidly to 50 percent of output at $6^{\circ} \mathrm{C}$ and $4^{\circ} \mathrm{C}$ respectively. ${ }^{36}$ The third is a more extreme version of these two damage functions where damages hit 50 percent of output at only $3^{\circ} \mathrm{C}$. Damages are subject to the same sequence of damage shocks with the parameters estimated in Section I.C. Since I am exploring outcomes when models are misspecified in the second part of the welfare analysis, I must simulate the model and calculate the welfare outcomes over a finite horizon. The frameworks' value functions only yield the true ex ante expected welfare when the model is specified correctly.

Table 3 displays ex ante welfare results for each of the frameworks relative to the uncertainty framework when the policymaker has correctly specified her damage model. The first line displays the lump sum present value benefit of using one of the frameworks over the uncertainty framework, the second

rise quicker will reduce the variance of beliefs.

${ }^{35}$ Recall that the robust control framework does not distort the actual state transitions, only the policymaker's beliefs about them.

${ }^{36}$ Except for very high levels of warming, the DICE damage function implies less damage than a damage function with parameters given by the distributional means associated with Table 1. 
Table 3: Measures of ex ante welfare of the learning, robust control, and robust control and learning frameworks relative to the uncertainty framework.

\begin{tabular}{lccc}
\hline Framework & Learning & Robust Control & Robust Control + Learning \\
\hline 2005 Lump Sum (billions of \$) & 758 & -156 & 615 \\
2005 Per Capita (\$) & 116 & -24 & 94 \\
BGE Gain (percent) & 0.039 & -0.008 & 0.032 \\
\hline
\end{tabular}

line displays the present value benefit in per capita terms, and the final line shows this in terms of balanced growth equivalent (BGE) consumption gain (Mirrlees and Stern, 1972; Lemoine and Traeger, 2014; Jensen and Traeger, 2016). The BGE is the difference in growth rates between two counterfactual consumption trajectories that grow at a constant rate and also yield the same welfare as the uncertainty framework and the comparison framework.

When damages are specified correctly, learning is worth $\$ 758$ billion, a one time payment in 2005 of $\$ 116$ per person, or a permanent gain in consumption of 0.039 percent. These welfare gains come about because the policymaker uses observations of damages to better match her policy to the actual damage function she faces. When the policymaker uses robust control but the damage function is correctly specified, she incurs losses of $\$ 156$ billion or equivalently $\$ 24$ per capita. Robust control induces the policymaker to use too high of a carbon tax which results in less consumption and lower welfare. Below I explore outcomes where the damage function is misspecified since that is the motivating factor for using robust control. The $\mathrm{RC}+\mathrm{L}$ framework yields a present value benefit of $\$ 615$ billion, 20 percent less than without robust control.

Figure 8 displays the present value ex post welfare gain for each of the frameworks relative to the uncertainty framework for four different time horizons. I compute ex post welfare for each combination of framework, damage function, and time horizon by first performing 10,000 simulations, each with a randomly drawn vector of annual damage shocks. I then average the sum of the present value of flow utilities across the 10,000 simulations. Finally I translate the expected present value from utils into dollars using the initial marginal 
utility of consumption from the uncertainty framework's optimal trajectory.

The horizontal axis on each plot in Figure 8 shows the four different time horizons over which ex post welfare is evaluated: 50, 100, 150, and 200 years. The vertical axis shows the present value expected welfare gain relative to the uncertainty framework. The uncertainty framework is omitted since it is the baseline framework. An equivalent level of welfare as the uncertainty framework is denoted by the dashed line. Plots above the dashed line indicate gains relative to the uncertainty framework and plots below the dashed line indicate losses. Each plot corresponds to one of the three catastrophic damage functions. All three catastrophic damage functions have the following form

$$
D\left(T_{t+1}^{s}, \omega_{t+1}\right)=\left(d_{1}\left[T_{t+1}^{s}\right]^{d_{2}}+d_{3}\left[T_{t+1}^{s}\right]^{d_{4}}\right) \omega_{t+1}
$$

The damage functions have the following common parameter values: $d_{1}=$ $.00284, d_{2}=2, d_{4}=6.754$. For the Weitzman damage function $d_{3}=5.07 \times$ $10^{-6}$, for the Dietz and Stern damage function $d_{3}=8.19 \times 10^{-5}$ and for the extreme damage function $d_{3}=5.85 \times 10^{-4}$. $\omega_{t+1}$ is the damage shock drawn from the same distribution as before.

The left panel displays outcomes when the policymaker's damage model is misspecified and the true damage function is the one developed in Weitzman (2012). Learning (with or without robust control) delivers ex post welfare gains over time horizons of 50-150 years. Although the policymaker has misspecified her model by omitting the extra catastrophic term, and she mis-learns by using the information from new damage observations to update $d_{2}$ instead of changing the structure of her damage function, learning a misspecified damage function improves ex post welfare for a set of time horizons. Until temperature rises substantially more than the initial level, the Weitzman damage function results in relatively low damages. The policymaker believes this is because she faces a low true value of $d_{2}$ and revises her expectation down to about 1.28 in 2105 when temperature is only $2.22^{\circ} \mathrm{C}$. Despite the damage function misspecification, this helps her push policy in the right direction for the near-term low damage world and she only sets a carbon tax of $\$ 32 / \mathrm{tCO}_{2}$ in 2105 . Welfare 


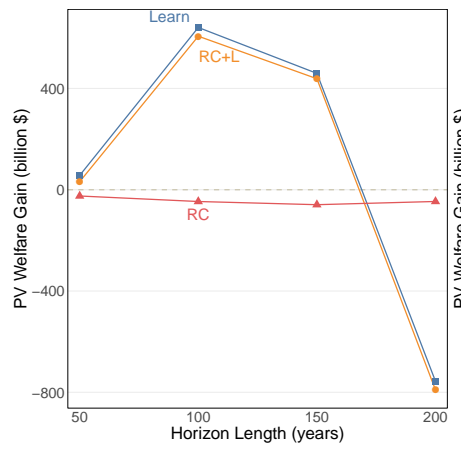

(a) Weitzman

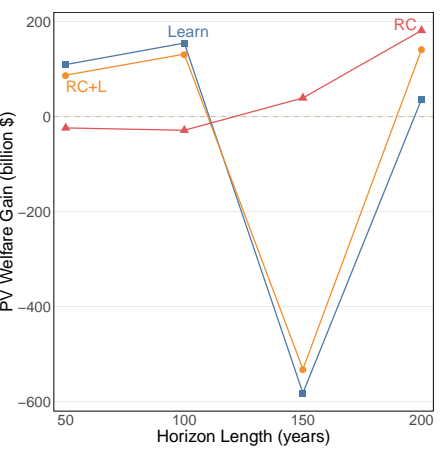

(b) Dietz and Stern

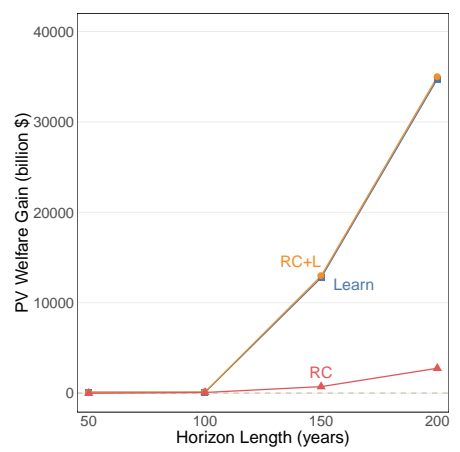

(c) Extreme

Figure 8: The present value welfare gain of the learning, robust control, and robust control and learning frameworks relative to the uncertainty framework for three different catastrophic damage functions. The left panel is for the Weitzman (2012) damage function where damages reach 50 percent of GDP at $6^{\circ} \mathrm{C}$, the center panel is for the Dietz and Stern (2015) damage function where damages reach 50 percent of GDP at $4^{\circ} \mathrm{C}$ and the right panel is for an extreme damage function where damages reach 50 percent of GDP at $3^{\circ} \mathrm{C}$. Ex post welfare gains are averaged over 10,000 simulations, each with a random sample of damage shocks $\left\{\omega_{2005}, \ldots, \omega_{2105}\right\}$.

gains are over $\$ 400$ billion for 100 and 150 year time horizons. Over time, this low carbon tax results in accelerated warming and eventually, and unexpectedly to the policymaker because of her misspecification, massive damages. Damages are nearly 4 times the levels of the uncertainty framework in the last 50 years of the simulation, and consumption under the learning framework is lower than under the uncertainty framework by tens of trillions of dollars per year. The accumulation of damage more than offsets the early welfare gains and generates total losses of over $\$ 750$ billion in present value terms by 2205 .

The robust control framework results in ex post welfare losses of up to $\$ 60$ billion even out to 200 years in the future. This is because when a non-learning policymaker is optimizing carbon taxes under her misspecified damage function, temperature never crosses $3^{\circ} \mathrm{C}$, so the catastrophic part of the Weitzman (2012) damage function does not kick in. Over time horizons up to 200 years, the robust control policymaker implements too stringent of a carbon tax relative to the uncertainty framework. 
The middle panel shows welfare outcomes when the true damage function is the one developed in Dietz and Stern (2015). Here learning results gains of about $\$ 100$ billion relative to the uncertainty framework over the first 100 years, large losses at 150 years, and small gains at 200 years. Similar to the left panel, the policymaker believes early on that she is not in a high damage world because temperature does not reach the point where the catastrophic part of the damage function kicks in. Near the end of the first century, she believes $d_{2}$ to be as low as 1.72. Correspondingly, she sets a moderate carbon tax of $\$ 43 / \mathrm{tCO}_{2}$ in 2075 as opposed to a tax of $\$ 54 / \mathrm{tCO}_{2}$ under the uncertainty framework, and she reallocates output toward consumption which increases present value ex post welfare at a 100 year horizon. In the second century, temperature crosses $2^{\circ} \mathrm{C}$ and gets closer to the catastrophic part of the damage function. The policymaker begins revising her beliefs upward and by 2155 she expects $d_{2}$ to be 2.30 . This rapid shift in her beliefs leads to a reallocation of output from consumption toward abatement. The learning carbon tax triples over a 50 year time span, from $\$ 79 / \mathrm{tCO}_{2}$ in 2105 to $\$ 255 / \mathrm{tCO}_{2}$ in 2155 , while under the uncertainty framework the carbon tax only about doubles over this timespan, from $\$ 87 / \mathrm{tCO}_{2}$ to $\$ 159 / \mathrm{tCO}_{2}$. This aggressive climate action results in short term welfare losses between 2105 and 2155, but it improves ex post welfare relative to the uncertainty framework by 2205 through reduced warming and damages. Adding robust control on top of learning increases welfare by about $\$ 100$ billion in 2205 relative to learning alone because of more aggressive climate action.

When using robust control but not learning, positive net benefits can be achieved relative to the uncertainty framework after 150 years. Expected ex post welfare gains are $\$ 180$ billion at 200 years in the future. Over the first century, temperature is below $2^{\circ} \mathrm{C}$ and the Dietz and Stern (2015) damage function closely follows the actual DICE damage function. Over this timeframe guarding against misspecification results in a carbon tax that's too high for a non-catastrophic damage function and slight welfare losses. After 150 years, temperature crosses $2.5^{\circ} \mathrm{C}$ for both the robust control and uncertainty frameworks, and damages start growing quicker as temperature begins reach- 
ing catastrophic levels. By the end of the second century, the avoided damages from the robust control framework's more aggressive carbon tax preserves an additional half a percentage point of output per year.

The right panel shows welfare outcomes under the extreme damage function where damages reach 50 percent of output at only $3^{\circ} \mathrm{C}$. Here learning delivers large ex post welfare gains after the first century. The catastrophic part of the damage function kicks in rapidly and the learning policymaker quickly adapts her carbon tax in the right direction despite her misspecified damage function. At the end of the first century, the learning and $\mathrm{RC}+\mathrm{L}$ policymakers set carbon taxes over twice as high as under the uncertainty framework and have nearly achieved full abatement of carbon emissions. By the end of the second century the learning and $\mathrm{RC}+\mathrm{L}$ carbon taxes are 8 times as large. Learning achieves welfare gains of up to $\$ 35$ trillion dollars after 200 years. Using robust control alone also begins delivering positive welfare gains at around 100 years. At this

point the robust control tax is only about 5 percent larger than the uncertainty tax. After 200 years, robust control welfare gains are $\$ 3$ trillion and the robust control tax is now 15 percent larger. Learning welfare gains are an order of magnitude larger than robust control welfare gains.

\section{Conclusions}

Most economists believe that damage functions in IAMs are misspecified. Yet, analyses of optimal climate policy have not investigated the effects of endogenously improving the damage function over time, or for adapting policy to take account of the widely noted concerns about damage function misspecification. I fill this gap in the literature by comparing the performance of four policy frameworks with different degrees of damage assumptions. The uncertainty framework accounts for uncertainty over all parameters of the damage function. The learning framework acknowledges that economists do learn over time and allows the policymaker to endogenously update her distribution over the uncertain temperature elasticity of damages. The robust control framework borrows techniques from the macroeconomic literature to incorporate concerns 
that the damage function is misspecified in unknown ways. The robust control policymaker constructs policies that guard against model misspecification. Last, the robust control and learning framework combines concern for model misspecification with updating of beliefs over the temperature elasticity of damages. For each of the frameworks, I demonstrate how misspecification concerns, and uncertainty about damages and future beliefs feed into the optimal carbon tax.

I find that uncertainty over the damage parameters generally increases the optimal carbon tax through the state uncertainty adjustment. A decomposition of the carbon tax into six uncertainty-related channels reveals that there are conflicting effects of uncertainty on the optimal carbon tax due to precaution and insurance motives. Depending on whether the policymaker learns about the damage function, motives to insure against model misspecification may increase or decrease the carbon tax, but the total effect of robust control is to increase the carbon tax.

Learning about the temperature elasticity of damages can deliver ex ante welfare gains worth hundreds of billions of dollars, indicating that current research aimed at updating damage functions has the potential to be extremely valuable. Ex ante welfare losses from using robust control when the model has been correctly specified are $\$ 156$ billion. In cases where the model's damage function is misspecified and the true damage function instead allows for catastrophe at temperatures of $3^{\circ} \mathrm{C}$ or higher, robust control and (incorrect) learning can both achieve higher welfare than accounting for parametric damage uncertainty alone, but with several caveats. Learning and robust control both perform well when the damage function has been highly misspecified, such as in worst-case scenarios where massive damages occur at temperatures we are expected to reach well before the end of the century along a business as usual trajectory. However if damages are expected to be low and noncatastrophic until $4{ }^{\circ} \mathrm{C}$ or higher, this may not be true. Learning about a misspecified damage function can backfire and reduce welfare by erroneously ruling out that there is catastrophe further down the line.

This paper's results make several contributions to the broader discussions 
about the specification of damage functions and the updating of damage functions. ${ }^{37}$ The first is to raise the question about whether a focus on protecting against Weitzman (2012) and Dietz and Stern (2015) style catastrophe is of first-order importance since the effects of a common method to guard against misspecification are small relative to that of learning. One omitted factor in my analysis is that uncertainty about climate sensitivity should make these catastrophes more relevant, however I leave adding climate sensitivity uncertainty into this framework to future work. ${ }^{38}$ The second contribution is to emphasize the high stakes of current research on estimating damages. The welfare effects of updating beliefs are large, and their magnitude and sign depend on the form of the true damage function. The damage functional form onto which updated estimates are placed will have a major influence on the real world effectiveness of IAM policy prescriptions.

The sensitivity of welfare to damage functional forms suggests that damage functions must simultaneously be more flexible and also better grounded in science in order to improve the utility of IAMs for policymaking. Continually updating quadratic or even general monomial damage functions will not adequately capture non-linearities. However, in order to estimate future damages under temperatures never before seen in human history, we cannot simply rely on flexible polynomial forms and statistical updating. Future temperatures will be far outside our historical sample and extrapolating without any structure on the damage function will give us poor estimates of future damages. More scientific guidance is needed to tell modelers what damage observations today might mean for the structure of the real world damage function at never before seen temperatures. The dynamic stochastic IAM literature has begun making inroads in using scientific knowledge to better incorporate tipping points into models (e.g. Lemoine and Traeger, 2014, 2016b; Cai, Judd and Lontzek, 2018), but there is much work left to be done to address other

\footnotetext{
${ }^{37}$ Beyond the greater damage function debate, this paper also points out the relevance of inter-generational equity. The costs of a more robust climate policy will be borne by current generations but the benefits will not be felt until potentially hundreds of years in the future.

${ }^{38}$ Future work may also focus on learning about both parameters of the damage function or modeling learning in a way that is more agnostic about the damage functional form.
} 
areas of the climate-economy system. 


\section{References}

Ackerman, F, and Elizabeth A. Stanton. 2012. "Climate Risks and Carbon Prices: Revising the Social Cost of Carbon." Economics : The OpenAccess, Open-Assessment E-Journal, 6(10).

Ackerman, Frank, Elizabeth A. Stanton, and Ramón Bueno. 2010. "Fat Tails, Exponents, Extreme Uncertainty: Simulating Catastrophe in DICE." Ecological Economics, 69(8): 1657-1665.

Anderson, Evan W., William A. Brock, Lars Peter Hansen, and Alan H. Sanstad. 2014. "Robust Analytical and Computational Explorations of Coupled Economic-Climate Models with Carbon-Climate Response."

Athanassoglou, Stergios, and Anastasios Xepapadeas. 2012. "Pollution Control with Uncertain Stock Dynamics: When, and How, to be Precautious." Journal of Environmental Economics and Management, 63(3): 304320.

Barrage, Lint. Forthcoming. "Optimal Dynamic Carbon Taxes in a ClimateEconomy Model with Distortionary Fiscal Policy." The Review of Economic Studies.

Berger, Loic, and Massimo Marinacci. 2017. "Model Uncertainty in Climate Change Economics." , (Cmcc): 1-33.

Berger, Loïc, Johannes Emmerling, and Massimo Tavoni. 2016. "Managing catastrophic climate risks under model uncertainty aversion." Management Science, 63(3): 749-765.

Brock, William, and Anastasios Xepapadeas. 2017. "Climate change policy under polar amplification." European Economic Review, 99: 93-112.

Burke, Marshall, Solomon M Hsiang, and Edward Miguel. 2015. "Climate and Conflict." Annual Review of Economics, 7(1): 577-617. 
Cai, Yongyang, Kenneth L. Judd, and Thomas S. Lontzek. 2018. "The Social Cost of Carbon with Economic and Climate Risks." Journal of Political Economy.

Cai, Yongyang, Kenneth L Judd, Timothy M Lenton, Thomas S Lontzek, and Daiju Narita. 2015. "Environmental Tipping Points Significantly Affect the Cost-Benefit Assessment of Climate Policies." Proceedings of the National Academy of Sciences, 112(15): 4606-4611.

Cogley, Timothy, Riccardo Colacito, Lars Peter Hansen, and Thomas J. Sargent. 2008. "Robustness and U.S. Monetary Policy Experimentation." Journal of Money, Credit and Banking, 40(8): 1599-1623.

Crost, Benjamin, and Christian P. Traeger. 2013. "Optimal Climate Policy: Uncertainty Versus Monte Carlo." Economics Letters, 120(3): 552558.

Crost, Benjamin, and Christian P. Traeger. 2014. "Optimal CO2 Mitigation Under Damage Risk Valuation." Nature Climate Change, 4(7): 631-636.

Dell, Melissa, Benjamin F Jones, and Benjamin A Olken. 2012. "Temperature Shocks and Economic Growth: Evidence from the Last Half Century." American Economic Journal: Macroeconomics, 4(3): 66-95.

Diaz, Delavane B., and Frances C. Moore. 2017. "Quantifying the Economic Risks of Climate Change." Nature Climate Change, 7(11): 774-782.

Dietz, Simon, and Frank Venmans. 2019. "Cumulative carbon emissions and economic policy: in search of general principles." Journal of Environmental Economics and Management, 96: 108-129.

Dietz, Simon, and Nicholas Stern. 2015. "Endogenous Growth, Convexity of Damage and Climate Risk: How Nordhaus' Framework Supports Deep Cuts in Carbon Emissions." Economic Journal, 125(583): 574-620.

Dreze, Jacques H., and Franco Modigliani. 1972. "Consumption Decisions Under Uncertainty." Journal of Economic Theory, 5(3): 308-335. 
Fitzpatrick, Luke G, and David L Kelly. 2017. "Probabilistic Stabilization Targets." Journal of the Association of Environmental and Resource Economists, 4(2): 611-657.

Gilboa, I, and D Schmeidler. 1989. "Maxmin Expected Utility with NonUnique Prior." Journal of Mathematical Economics, 18: 141-153.

Gollier, Christian. 2004. The Economics of Risk and Time. MIT press.

Gollier, Christian. 2010. "Ecological Discounting." Journal of Economic Theory, 145(2): 812-829.

Gonzalez, Fidel. 2007. "Precautionary Principle and Robustness for a Stock Pollutant with Multiplicative Risk." Environmental and Resource Economics, 41(1): 25-46.

Greenstone, M., E. Kopits, and A. Wolverton. 2013. "Developing a Social Cost of Carbon for US Regulatory Analysis: A Methodology and Interpretation." Review of Environmental Economics and Policy, 7(1): 2346.

Hanemann, WM. 2008. "What is the Economic Cost of Climate Change?" CUDARE Working Papers.

Hansen, Lars Peter, and Thomas J. Sargent. 2007. "Recursive Robust Estimation and Control Without Commitment." Journal of Economic Theory, 136(1): 1-27.

Hansen, Lars Peter, and Thomas J. Sargent. 2008. Robustness. Princeton, N.J.:Princeton University Press.

Heutel, Garth, Juan Moreno-Cruz, and Soheil Shayegh. 2016. "Climate Tipping Points and Solar Geoengineering." Journal of Economic Behavior \& Organization, 132: 19-45.

Heutel, Garth, Juan Moreno-Cruz, and Soheil Shayegh. 2018. "Solar Geoengineering, Uncertainty, and the Price of Carbon." Journal of Environmental Economics and Management, 87: 24-41. 
Hope, Chris. 2006. "The Marginal Impact of CO2 from PAGE2002: An Integrated Assessment Model Incorporating the IPCC's Five Reasons for Concern." Integrated Assessment, 6: 19-56.

Howard, Peter. 2014. "Omitted Damages: What's Missing from the Social Cost of Carbon."

Howard, Peter H, and Derek Sylvan. 2016. "The Wisdom of the Economic Crowd: Calibrating Integrated Assessment Models Using Consensus."

Howard, Peter H, and Thomas Sterner. 2017. "Few and not so far between: a meta-analysis of climate damage estimates." Environmental and Resource Economics, 68(1): 197-225. doi:10.1007/s10640-017-0166-z.

Hsiang, Solomon M. 2016. Annual Review of Resource Economics, 8: 43-75.

Hsiang, Solomon, Michael Delgado, Shashank Mohan, D J Rasmussen, Robert Muirwood, Paul Wilson, Michael Oppenheimer, Kate Larsen, and Trevor Houser. 2017. "Estimating Economic Damage from Climate Change in the United States." Science, 1369(June): 1362-1369.

Hsiang, Solomon, Paulina Oliva, and Reed Walker. 2019. "The distribution of environmental damages." Review of Environmental Economics and Policy, 13(1): 83-103.

Hwang, In Chang, Frédéric Reynès, and Richard SJ Tol. 2017. "The effect of learning on climate policy under fat-tailed risk." Resource and Energy Economics, 48: 1-18.

IPCC. 2013. Climate Change 2013: The Physical Science Basis: Working Group I Contribution to the Fifth Assessment Report of the Intergovernmental Panel on Climate Change. Cambridge University Press.

Jensen, Svenn, and CP Traeger. 2016. "Pricing Climate Risk."

Kelly, David L., and Charles D. Kolstad. 1999. "Bayesian Learning, Growth, and Pollution." Journal of Economic Dynamics and Control, 23: $491-518$. 
Kelly, David L, and Zhuo Tan. 2015. "Learning and Climate Feedbacks: Optimal Climate Insurance and Fat Tails." Journal of Environmental Economics and Management, 72: 98-122.

Kimball, Miles S. 1990. "Precautionary Saving in the Small and in the Large." Econometrica, 58(1): 53-73.

Knutti, Reto, Maria A. A. Rugenstein, and Gabriele C. Hegerl. 2017. "Beyond equilibrium climate sensitivity." Nature Geoscience, 10(10): 727.

Kopp, Robert E, Alexander Golub, Nathaniel O Keohane, and Chikara Onda. 2012. "The Influence of the Specification of Climate Change Damages on the Social Cost of Carbon." Economics: The OpenAccess, Open-Assessment E-Journal, 6.

Kriegler, Elmar, Jim W Hall, Hermann Held, Richard Dawson, and Hans Joachim Schellnhuber. 2009. "Imprecise probability assessment of tipping points in the climate system." Proceedings of the national Academy of Sciences, 106(13): 5041-5046.

Leach, Andrew. 2007. "The Climate Change Learning Curve." Journal of Economic Dynamics and Control, 31(5): 1728-1752.

Leland, Hayne E. 1968. "Saving and Uncertainty: The Precautionary Demand For Saving." The Quarterly Journal of Economics, 82(3): 465-473.

Lemoine, Derek, and Christian Traeger. 2014. "Watch Your Step: Optimal Policy in a Tipping Climate." American Economic Journal: Economic Policy, 6(1): 137-166.

Lemoine, Derek, and Christian Traeger. 2016a. "Ambiguous Tipping Points." Journal of Economic Behavior \&3 Organization.

Lemoine, Derek, and Christian Traeger. 2016b. "Economics of Tipping the Climate Dominoes." Nature Climate Change, 6(5): 514-519. 
Lemoine, Derek, and Haewon C McJeon. 2013. "Trapped Between Two Tails: Trading Off Scientific Uncertainties via Climate Targets." Environmental Research Letters, 8(3).

Lemoine, Derek, and Ivan Rudik. 2017. "Managing Climate Change Under Uncertainty: Recursive Integrated Assessment at an Inflection Point." Annual Review of Resource Economics, 9.

Li, Xin, Borghan Narajabad, and Ted Temzelides. 2016. "Robust Dynamic Optimal Taxation and Environmental Externalities."

Lontzek, Thomas S., Yongyang Cai, Kenneth L. Judd, and Timothy M. Lenton. 2015. "Stochastic Integrated Assessment of Climate Tipping Points Indicates the Need for Strict Climate Policy." Nature Climate Change, 5(5): 441-444.

Matthews, H Damon, Nathan P Gillett, Peter a Stott, and Kirsten Zickfeld. 2009. "The proportionality of global warming to cumulative carbon emissions." Nature, 459(7248): 829-32.

Mirrlees, James A, and Nicholas H Stern. 1972. "Fairly good plans." Journal of Economic Theory, 4(2): 268-288.

Moore, Frances C., and Delavane B. Diaz. 2015. "Temperature Impacts on Economic Growth Warrant Stringent Mitigation Policy." Nature Climate Change, , (January): 1-5.

National Academy of Sciences. 2017. Valuing Climate Damages: Updating Estimation of the Social Cost of Carbon Dioxide.

Nordhaus, W, and Paul Sztorc. 2013. "DICE 2013R: Introduction and User's Manual."

Nordhaus, William D. 2008. A Question of Balance: Weighing the Options on Global Warming Policies. New Haven:Yale University Press. 
Pindyck, Robert S. 2012. "Uncertain Outcomes and Climate Change Policy." Journal of Environmental Economics and Management, 63(3): 289303.

Pindyck, Robert S. 2013. "Climate Change Policy: What Do the Models Tell Us?" Journal of Economic Literature, 51(3): 860-872.

Pindyck, Robert S. 2017. "The use and misuse of models for climate policy." Review of Environmental Economics and Policy, 11(1): 100-114.

Roseta-Palma, C, and A Xepapadeas. 2004. "Robust Control in Water Management." Journal of Risk and Uncertainty, 29(1): 21-34.

Sherwood, Steven C, and Matthew Huber. 2010. "An adaptability limit to climate change due to heat stress." Proceedings of the National Academy of Sciences, 107(21): 9552-9555.

Stanton, Elizabeth A., Frank Ackerman, and Sivan Kartha. 2009. "Inside the Integrated Assessment Models: Four Issues in Climate Economics." Climate and Development, 1(2): 166.

Sterner, T., and U. M. Persson. 2008. "An Even Sterner Review: Introducing Relative Prices into the Discounting Debate." Review of Environmental Economics and Policy, 2(1): 61-76.

Stern, Nicholas. 2006. "The Stern Review on the Economic Effects of Climate Change."

Stoerk, Thomas, Gernot Wagner, and Robert ET Ward. 2018. "Policy BriefRecommendations for Improving the Treatment of Risk and Uncertainty in Economic Estimates of Climate Impacts in the Sixth Intergovernmental Panel on Climate Change Assessment Report." Review of Environmental Economics and Policy, 12(2): 371-376.

Tol, Richard S. J. 2009. "The Economic Effects of Climate Change." The Journal of Economic Perspectives, 23(2): 29-51. 
Weitzman, Martin. 2010. "Some Dynamic Economic Consequences of the Climate Sensitivity Inference Dilemma." Handbook of Environmental Accounting.

Weitzman, Martin L. 2012. "GHG Targets as Insurance Against Catastrophic Climate Damages." Journal of Public Economic Theory, 14(2): 221244 . 


\section{Online Appendix: Optimal Climate Policy when Damages are Unknown - Ivan Rudik}

The online appendix gives full details on the dynamic stochastic climate-economy model, describes how I use a linear climate model consistent with recent developments in climate science, describes my computational algorithms, provides an error analysis of the results, shows the full derivation of the carbon tax, and plots the certainty tax.

\section{A The Full Stochastic Climate-Economy Model}

The model is a Ramsey-Cass-Koopmans growth model coupled to a climate system. The model is governed by a representative policymaker whose objective is to maximize her expected discounted welfare. Each period lasts one year and the model begins in 2005.

In each period $t$, the policymaker has an endowment of capital $K_{t}$, labor $L_{t}$, and technology $A_{t}$. To improve numerical accuracy I express capital in effective labor terms, $k_{t}=\frac{K_{t}}{A_{t} L_{t}}$ (Traeger, 2014). Capital, labor and technology are combined in a Cobb-Douglas production function to produce gross output

$$
Y_{t}^{g}=k_{t}^{\kappa t}
$$

Warming of the Earth's surface causes damage to output, resulting in net output after damages

$$
Y_{t}^{n}=Y_{t}^{g} \mathcal{L}_{t}
$$

where $\mathcal{L}_{t}$ is the fraction of output remaining after damages as defined in the main text (fractional net output)

$$
\mathcal{L}_{t}=\frac{1}{1+d_{1}\left[T_{t}^{s}\right]^{d_{2}} w_{t}}
$$

The policymaker has three ways to use her remaining output after damages. First, she can use it for consumption $C_{t}$, also expressed in effective labor terms $c_{t}=\frac{C_{t}}{A_{t} L_{t}}$ to 
increase flow utility

$$
U\left(c_{t}\right)=\frac{c_{t}^{1-\eta}}{(1-\eta)}, \quad \eta \neq 1 .
$$

Second, she can use it to abate some fraction $\alpha_{t} \in[0,1]$ of emissions from factor production with a cost given by

$$
C\left(\alpha_{t}\right)=\Psi_{t} \alpha_{t}^{a_{2}} .
$$

The residual output is left for investment into increasing the future capital stock, which depreciates at an annual rate of $\delta_{k}$. Net emissions after abatement $e_{t}$ is

$$
e_{t}=\sigma_{t}\left(1-\alpha_{t}\right) Y_{t}^{g}+B_{t} .
$$

$B_{t}$ is emissions from exogenous land use change, and $\sigma_{t}$ is the emissions intensity of output. Following the recent climate science literature (e.g. Matthews et al., 2009; IPCC, 2014; Knutti et al., 2017), I use a 1 state climate system that is a function of cumulative $\mathrm{CO}_{2}$ emissions. Contemporaneous surface temperature (relative to preindustrial) is proportional to cumulative $\mathrm{CO}_{2}$ emissions since preindustrial times

$$
T_{t}^{s}=\zeta \sum_{s=1880}^{t} e_{s},
$$

where $\zeta$ is the transient climate response to emissions (TCRE). Cumulative emissions has a Markov transition

$$
E_{t}=E_{t-1}+e_{t}
$$

so that I can write temperature as

$$
T_{t}^{s}=\zeta E_{t} .
$$

The model's exogenously evolving processes are

$$
\begin{aligned}
L_{t} & =L_{0}+\left(L_{\infty}-L_{0}\right)\left(1-\exp \left(-\delta_{L} t\right)\right) & & \text { (Labor population) } \\
g_{L, t} & =\delta_{L}\left(\frac{L_{\infty}}{L_{\infty}-L_{0}} \exp \left(\delta_{L} t\right)-1\right)^{-1} & & \text { (Labor growth rate) } \\
A_{t} & =A_{0} \exp \left(\left(1-\exp \left(-\delta_{A} t\right)\right) \frac{g_{A, 0}}{\delta_{A}}\right) & & \text { (Production technology) }
\end{aligned}
$$




$$
\begin{aligned}
g_{A, t} & =g_{A, 0} \exp \left(-\delta_{A} t\right) \\
\beta_{t} & =\exp \left(-\rho+(1-\eta) g_{A, t}+g_{L, t}\right) \\
\sigma_{t} & =\sigma_{0} \exp \left[\frac{g_{\sigma, 0}}{\delta_{\sigma}}\left(1-\exp \left(-\delta_{\sigma} t\right)\right)\right] \\
\Psi_{t} & =\frac{a_{0} \sigma_{t}}{a_{2}}\left(1-\frac{1-\exp \left(g_{\Psi} t\right)}{a_{1}}\right) \\
B_{t} & =B_{0} g_{B}^{t}
\end{aligned}
$$

(Production technology growth rate)

(Growth adjusted discount factor)

(Gross emissions per unit of output)

(Abatement cost coefficient)

(Non-industrial $\mathrm{CO}_{2}$ emissions)

Table A1 reports the values of the model parameters. The calibration of the distribution over $d_{1}, d_{2}$, and $\omega_{t+1}$ are described in the main text. 
Table A1: The parameters of the model.

\begin{tabular}{|c|c|c|}
\hline Parameter & Value & Description \\
\hline$A_{0}$ & 0.006 & Initial production technology \\
\hline$g_{A, 0}$ & 0.013 & Initial growth rate of production technology \\
\hline$\delta_{A}$ & 0.001 & Change in growth rate of production technology \\
\hline$L_{0}$ & 6514 & Year 2005 population (millions) \\
\hline$L_{\infty}$ & 8600 & Asymptotic population (millions) \\
\hline$\delta_{L}$ & 0.035 & Rate of approach to asymptotic population level \\
\hline$\sigma_{0}$ & 0.13 & Initial emission intensity of output (Gigatons of carbon per unit outpu \\
\hline$g_{\sigma, 0}$ & -0.0073 & Initial growth rate of decarbonization \\
\hline$\delta_{\sigma}$ & 0.003 & Change in growth rate of emissions intensity \\
\hline$a_{0}$ & 1.17 & Cost of backstop technology in 2005 ( $\$ 1000$ per ton of carbon) \\
\hline$a_{1}$ & 2 & Initial backstop technology cost / Final backstop technology cost \\
\hline$a_{2}$ & 2.8 & Abatement cost function exponent \\
\hline$g_{\Psi}$ & -0.005 & Growth rate of backstop technology cost \\
\hline$B_{0}$ & 1.1 & Initial non-industrial $\mathrm{CO}_{2}$ emissions (Gigatons of carbon) \\
\hline$g_{B}$ & -0.01 & Growth rate of non-industrial emissions \\
\hline$\kappa$ & 0.3 & Capital elasticity in production \\
\hline$\delta_{k}$ & 0.1 & Capital depreciation rate \\
\hline$E_{0}$ & 454.90 & Cumulative emissions in initial model year (Gigatons of carbon in 2005 \\
\hline$\rho$ & 0.015 & Pure rate of time preference \\
\hline$\eta$ & 2 & 1/EIS, and RRA \\
\hline$\zeta$ & 0.0016 & TCRE $\left({ }^{\circ} \mathrm{C}\right.$ per 1,000 gigatons of carbon $)$ \\
\hline$k_{0}$ & $137 /\left(A_{0} L_{0}\right)$ & Year 2005 effective capital \\
\hline$\mu_{c}$ & -5.38 & $d_{1}$ location parameter \\
\hline$\sigma_{c}^{2}$ & 0.38 & $d_{1}$ scale parameter \\
\hline $\exp \left(\mu_{c}+\frac{\sigma_{c}^{2}}{2}\right)$ & 0.00556 & $d_{1}$ mean \\
\hline $\exp \left(2 \mu_{c}+\sigma_{c}^{2}\right)\left[\exp \left(\sigma_{c}^{2}\right)-1\right]$ & $0.00379^{2}$ & $d_{1}$ variance \\
\hline$\mu_{0}$ & 1.88 & Year $2005 d_{2}$ mean \\
\hline$\Sigma_{0}$ & 0.20 & Year $2005 d_{2}$ variance \\
\hline$\mu_{\omega}$ & -0.59 & $\omega_{t}$ location parameter \\
\hline$\sigma_{\omega}^{2}$ & 1.18 & $\omega_{t}$ scale parameter \\
\hline $\exp \left(\mu_{\omega}+\frac{\sigma_{\omega}^{2}}{2}\right)$ & 1 & $\omega_{t}$ mean \\
\hline $\exp \left(2 \mu_{\omega}+\sigma_{\omega}^{2}\right)\left[\exp \left(\sigma_{\omega}^{2}\right)-1\right]$ & $1.22^{2}$ & $\omega_{t}$ variance \\
\hline$\mu_{c \omega}$ & -5.97 & Joint $d_{1}, \omega_{t}$ location parameter \\
\hline$\sigma_{c \omega}^{2}$ & 1.56 & Joint $d_{1}, \omega_{t}$ scale parameter \\
\hline
\end{tabular}


Without loss of generality, the robust control policymaker's problem is then

$$
\begin{aligned}
& V_{t}\left(k_{t}, E_{t}, \mathcal{L}_{t}, \mu_{t}, \Sigma_{t}\right)= \\
& \max _{c_{t}, \alpha_{t}}\left\{u\left(c_{t}\right)+\mathbb{E}_{d_{2}}\left[-\theta \log \left(\mathbb{E}_{d_{1}, \omega_{t+1}}\left[\exp \left(-\frac{\beta_{t} V_{t+1}\left(k_{t+1}, E_{t+1}, \mathcal{L}_{t+1}, \mu_{t+1}, \Sigma_{t+1}\right)}{\theta}\right)\right]\right)\right]\right\}
\end{aligned}
$$

subject to transitions:

$$
\begin{aligned}
k_{t+1} & =\exp \left(-\left(g_{L, t}+g_{A, t}\right)\right)\left[\left(1-\delta_{k}\right) k_{t}-c_{t}+\left(1-\Psi_{t} \alpha_{t}^{a_{2}}\right) Y_{t}^{g} \mathcal{L}_{t}\right], \\
E_{t+1} & =E_{t}+\sigma_{t}\left(1-\alpha_{t}\right) Y_{t}^{g}+B_{t}, \\
\mathcal{L}_{t+1} & =\frac{1}{1+d_{1}\left[\zeta E_{t+1}\right]^{d_{2}} \omega_{t+1}}, \\
\mu_{t+1} & =\frac{\left(\sigma_{w}^{2}+\sigma_{c}^{2}\right) \mu_{t}+\log \left(\zeta E_{t+1}\right) \Sigma_{t}\left[\log \left(\frac{1}{\mathcal{L}_{t+1}}-1\right)-\left(\mu_{c}+\mu_{w}\right)\right]}{\left(\sigma_{w}^{2}+\sigma_{c}^{2}\right)+\left[\log \left(\zeta E_{t+1}\right)\right]^{2} \Sigma_{t}}, \\
\Sigma_{t+1} & =\frac{\Sigma_{t}\left(\sigma_{w}^{2}+\sigma_{c}^{2}\right)}{\left(\sigma_{w}^{2}+\sigma_{c}^{2}\right)+\left[\log \left(\zeta E_{t+1}\right)\right]^{2} \Sigma_{t}} .
\end{aligned}
$$

Finally I constrain abatement to be less than 100 percent and I impose the resource constraint

$$
\begin{aligned}
& \alpha_{t} \leq 1 \\
& c_{t}+\Psi_{t} \alpha_{t}^{a_{2}} Y_{t}^{g} \mathcal{L}_{t} \leq Y_{t}^{g} \mathcal{L}_{t} .
\end{aligned}
$$

If the policymaker does not learn, then $\mu_{t+1}=\mu_{t}$ and $\Sigma_{t+1}=\Sigma_{t}$. Note that the transition for $\mu_{t+1}$ is stochastic and depends on the realization of $\omega_{t+1}$ and the true values of $d_{1}$ and $d_{2}$.

\section{A.1 Climate System}

To make the model tractable to solve numerically, I take advantage of recent findings in climate science that find warming is proportional to cumulative emissions of carbon (Matthews et al., 2009; IPCC, 2014; Knutti et al., 2017). This relationship comes about because a pulse of carbon into the atmosphere increases equilibrium temperature quickly by a constant amount (Matthews and Caldeira, 2008). This has lead scientists to conclude that contemporaneous temperature is almost entirely determined by the level of cumulative past emissions (Allen et al., 2009; Matthews et al., 2009). Past cumulative emissions is translated into temperature by a factor of 
proportionality called the transient climate response to emissions (TCRE).

Define the TCRE as (Williams et al., 2016; Dietz and Venmans, 2019)

$$
\zeta \equiv \frac{\Delta T}{\Delta E}=\frac{\Delta T}{\Delta M} \frac{\Delta M}{\Delta E}
$$

where $T$ is temperature, $M$ is atmospheric carbon, and $E$ is cumulative emissions. Let $\Delta$ denote changes since preindustrial levels. $\frac{\Delta T}{\Delta M}$ gives the change in warming from additional carbon in the atmosphere and $\frac{\Delta M}{\Delta E}$ gives the change in atmospheric carbon concentrations given a change in emissions. Each of these terms is individually non-linear but the product of this ends up being a simple proportional relationship for two key reasons: (1) warming from a pulse of emissions realizes quickly, and then is constant over time and (2) the marginal effect of emissions on temperature is constant and independent of the state of the climate.

How does this come about? ${ }^{1}$ First, $\frac{\Delta T}{\Delta M}$ is increasing and concave. Over time, greater $\mathrm{CO}_{2}$ concentrations lead to more warming as additional heat gets trapped. The relationship is concave because both the relationship between $\mathrm{CO}_{2}$ concentrations and temperature is approximately logarithmic, and the oceans' significant heat capacity leads to thermal inertia: a lag between changes in carbon concentrations and equilibrium warming. The second term, $\frac{\Delta M}{\Delta E}$, is increasing and convex. Additional emissions initially go into the atmosphere and increase atmospheric $\mathrm{CO}_{2}$, while some of these emissions eventually get absorbed in land and ocean carbon sinks. The convex relationship arises because the strength of these sinks is decreasing in cumulative emissions. If there has been greater past cumulative emissions, a larger fraction of the next emission will remain in the atmosphere instead of being absorbed by one of the sinks. The curvature in $\frac{\Delta T}{\Delta M}$ and $\frac{\Delta M}{\Delta E}$ effectively cancel out so that $\zeta$ is constant and positive.

Using the TCRE climate model allows me to reduce the climate system to 1 state as opposed to other options such as the 5 state DICE climate system. This makes using standard tensor product approximation methods tractable and updates the climate system to match the most recent developments in climate science. I use a TCRE value of $\zeta=0.0016\left({ }^{\circ} \mathrm{C} / 1,000\right.$ gigatons of carbon). This value and the proportional translation of cumulative emissions to temperature follow closely to parallel work on climate model uncertainty (Berger and Marinacci, 2017) and recent

\footnotetext{
${ }^{1}$ See Figure 1 of Millar et al. (2016) for plots of these relationships.
} 


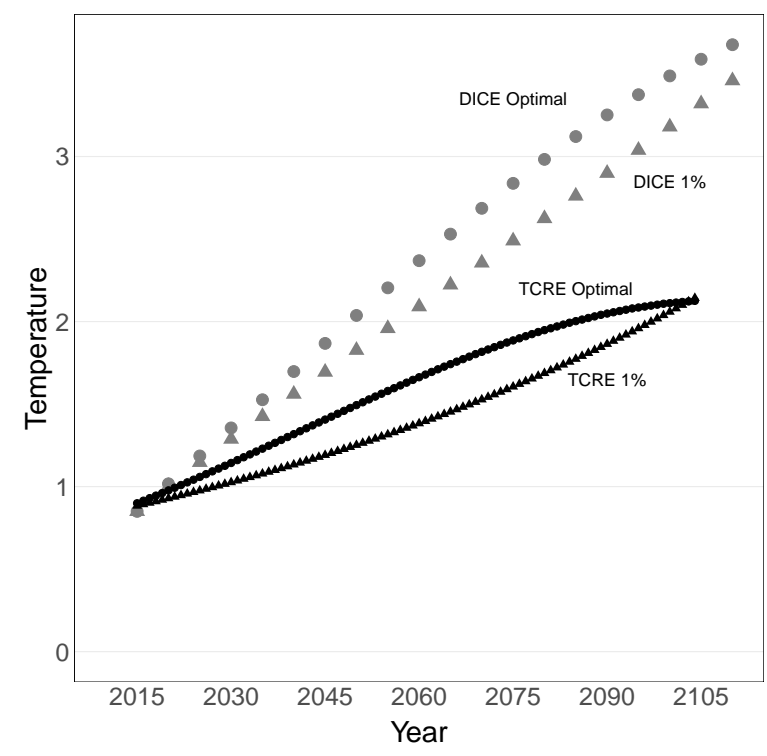

Figure A1: The temperature trajectories for DICE-2016 along the DICE-optimal emissions path (gray circles), DICE-2016 along an emissions path that grows cumulative emissions by $1 \%$ per year (gray triangles), the TCRE-based model used in this paper along the DICE-optimal emissions path (black circles) and the TCRE-based model used in the paper along an emissions path that grows cumulative emissions by $1 \%$ per year (black triangles).

climate economics papers (e.g. Dietz and Venmans, 2019; van der Ploeg, 2018). This value for the TCRE is in the middle of the most likely range estimated by the IPCC (IPCC, 2014; Knutti et al., 2017). $\zeta$ has been found to be constant up to various levels of cumulative emissions, including up to 2,000 GtC (Matthews et al., 2009), or 3,000 GtC (Leduc et al., 2015), and the TCRE has also been found to be approximately constant across all RCP scenarios (MacDougall and Friedlingstein, 2015).

Figure A1 plots temperature trajectories comparing the DICE climate system to the TCRE system. The DICE temperature trajectories are shown in gray and the TCRE trajectories are shown in black. Circle markers denote emissions trajectories that correspond to the optimal emissions trajectory from DICE-2016 while triangle markers correspond to an emissions trajectory that grows cumulative emissions by $1 \%$ per year. Both trajectories result in cumulative emissions of 1,300-1,400 GtC by 2105. The TCRE system predicts approximately $2.1^{\circ} \mathrm{C}$ of warming along these two trajectories which is well within the range of predicted temperatures of global climate models at this cumulative emissions level (IPCC, 2013). The DICE model predicts 
about $1^{\circ} \mathrm{C}$ more warming than the TCRE model and tends to be on the high end of temperature predictions from the suite of global climate models used in IPCC (2013).

\section{A.2 Model Solution Method and Error Analysis}

General approximation scheme The model is solved using value function iteration on a finite horizon. The collocation grid and polynomial interpolant are built using standard tensor product methods. The grid is constructed from a tensor product of one dimensional vectors of zeros of Chebyshev polynomials. For the learning frameworks, the grid has 2,100 grid points for 2005-2505 and 1,575 grid points for 2506-2605. ${ }^{2}$ For the non-learning frameworks, the grid has 729 grid points. ${ }^{3}$ The polynomial interpolant is constructed from a tensor product of Chebyshev polynomials. Below I test the sensitivity of my results to the number of collocation grid points, quadrature points, and the bounds for the state space.

Terminal value function The terminal year is 2605 . The terminal continuation value function corresponding to 2606 has the policymaker not learning while holding her initial beliefs about $\left\{d_{2}, d_{1}, \omega_{t+1}\right\}$, and has all exogenous processes held constant at their 2606 levels. Changing the terminal value function to one where the policymaker does not expect damage stochasticity and believes the damage function to be exactly equal to that in the conventional DICE model does not significantly alter the results since the continuation value 600 years in the future is effectively discounted to zero.

Expectations Expectations over future states are taken using Gauss-Hermite quadrature with 11 unique points over the $d_{2}$ distribution, and 11 unique points over the joint $\left\{d_{1}, \omega_{t+1}\right\}$ distribution for a total of 121 quadrature points. Note that the support of the location parameter for a lognormal distribution is the entire real line. Depending on the draws of the random variables, it can take on any value in $(-\infty, \infty)$. Below I test the sensitivity of my results to the number of quadrature points to determine whether evaluating the random variables at additional points further into the tail of the distribution significantly affects my results.

\footnotetext{
${ }^{2}$ These correspond to 5 unique grid points for cumulative emissions, 3 for the location parameter, 7 for the scale parameter, 5 for fractional net output, and either 3 or 4 for effective capital.

${ }^{3}$ This corresponds to 9 on each state.
} 
Table A2: Upper and lower bounds for each state. In the case of learning and $\mathrm{RC}+\mathrm{L}$ frameworks the bounds are only for the first iteration of the adaptive grid algorithm.

\begin{tabular}{lccccc}
\hline & $k$ & $E$ & $\mu$ & $\Sigma$ & $\mathcal{L}$ \\
\hline State Upper Bound: Uncertainty and RC & 4.30 & 3500.0 & - & - & 1.0 \\
State Lower Bound: Uncertainty and RC & 0.65 & 454.9 & - & - & 0.3 \\
Initial State Upper Bound: Learning and RC+L & 6.0 & 5000.0 & 3.13 & $.45^{2}$ & 1.0 \\
Initial State Lower Bound: Learning and RC+L & 0.0 & 454.9 & 0.63 & 0.5 & 0.4 \\
\hline
\end{tabular}

Adaptive grid I use a time-varying set of bounds to approximate the learning and $\mathrm{RC}+\mathrm{L}$ value functions. ${ }^{4}$ Earlier years have tighter bounds since the set of possible realized states is smaller, while later years have wider bounds. ${ }^{5}$ This allows me to use significantly fewer collocation points than on a standard hyperrectangle grid. I generate this adaptive grid using the following algorithm:

1. Solve the model on the time-invariant set of bounds given by Table A2. Use 3 unique grid points for effective capital, 5 for cumulative emissions, 3 for the location parameter, 7 for the scale parameter, and 5 for fractional net output.

2. Perform 1,000 simulations that are 301 years in length.

3. For each state

(a) Recover the maximum and minimum simulated values in each year over the 1,000 simulations.

(b) These time series will be noisy in later years even with a very large number of simulations, so fit a spline to the time series of maxima and another spline to the time series of minima. ${ }^{6}$

(c) Set the new time $t$ upper/lower bounds to a be weighted average of the fitted maxima/minima splines evaluated at time $t$, and the previous time

\footnotetext{
${ }^{4}$ This scheme is similar to that used in a number of recent stochastic implementations of DICE with even more states (Cai et al., 2015; Lontzek et al., 2015; Cai et al., 2016, Forthcoming). Cai (2018) provides more details on why this approach is particularly efficient for climate-economy models related to DICE and how it can allow researchers to solve extremely complicated models in minutes.

${ }^{5}$ For example, cumulative emissions can only grow so much in the first five years and the trajectory is non-decreasing since there are no negative emissions technologies.

${ }^{6}$ Smoothing out a time series of maxima or minima takes many more simulation runs than smoothing out the mean. The process can be greatly accelerated by fitting a spline.
} 
$t$ bounds, where weights on the bounds given by the splines are shown in Table A3. ${ }^{7}$

4. Solve the model on the new time-varying set of bounds obtained in Step 3. Use 3 unique grid points for effective capital, 5 for cumulative emissions, 3 for the location parameter, 7 for the scale parameter, and 5 for fractional net output.

5. Repeat steps 2-4 16 times.

6. Repeat steps 2-4 4 times but use 5 grid points for effective capital starting at year 2505 .

7. Set the bounds in 2005 to effectively be an epsilon ball around the initial state since it is the only state reached in that year.

8. Solve the model on this final set of adapted bounds using 4 unique grid points for effective capital, 5 for cumulative emissions, 3 for the location parameter, 7 for the scale parameter, 5 for fractional net output.

I do not adapt the bounds for the scale parameter (i.e. the 0.0 weights in Table A3) because the misspecified damage function simulations tend to result in it jumping outside the bounds if its bounds are adapted, while the other states stay within the bounds even when the damage function is misspecified. I do not adapt the upper bound for fractional net output because the maximum simulated values are generally very close to 1.0, the initial upper bound. The scale parameter bounds are not adapted except for the initial period. The algorithm allows for tight bounds on the remaining states compared to the non-learning frameworks' hyperrectangle grid.

Figure A2 depicts the bounds and 1,000 simulated state trajectories. The final set of adapted grid bounds are the thick black lines, the simulated state trajectories are the thin gray lines, and the expected state trajectories are the dotted black lines. For the states where the bounds were adapted, the bounds are tight around the set of states actually reached in simulations. For about 20 total simulation years each, fractional net output and the location parameter are no longer within the state bounds where the value function approximant is reliable. Although this introduces error, 20 years outside the bounds is a negligible fraction of the 101,000 total years displayed in

\footnotetext{
${ }^{7}$ This is similar to damping procedures used in other iterative algorithms like infinite-horizon value function iteration.
} 


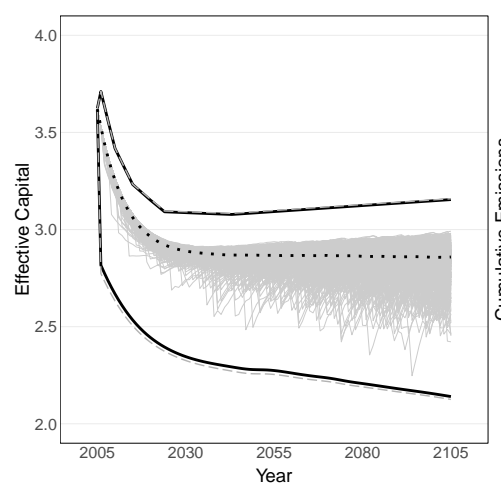

(a) Effective Capital

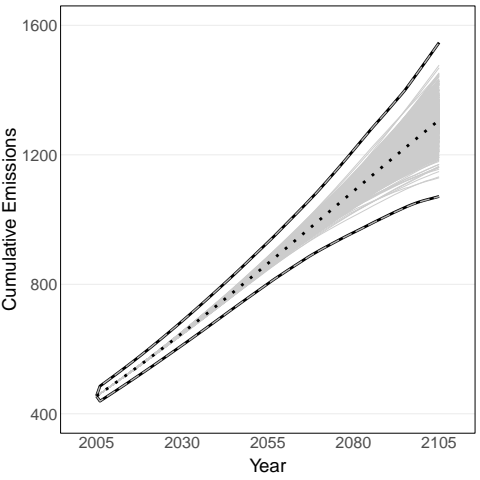

(b) Cumulative Emissions

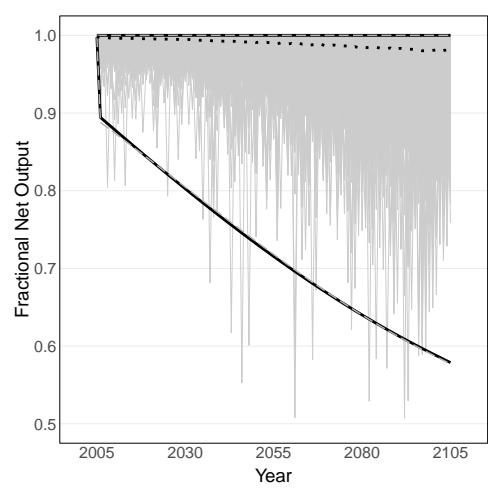

(c) Fractional Net Output

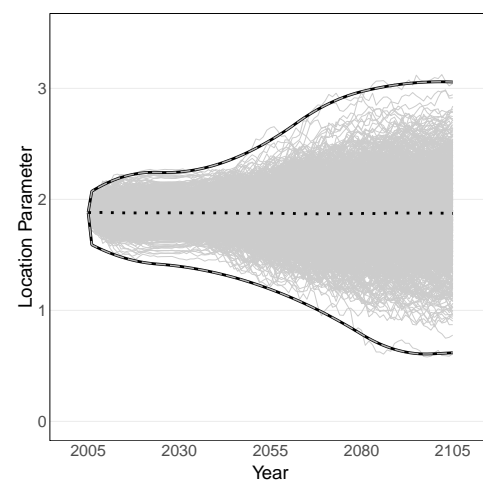

(d) Location Parameter

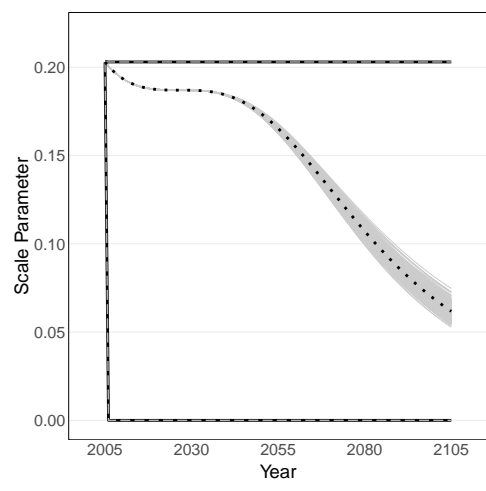

(e) Scale Parameter

Figure A2: The bounds for each state for the learning framework and the robust control and learning framework (thick black line). The bounds for each state for the learning framework and the robust control and learning framework in the secondto-last iteration of the adaptive grid algorithm (thick dashed gray line). Simulated state trajectories from the first 1,000 simulations (thin gray lines). Expected state trajectory (dotted black line).

the simulations (101 year simulation $\times 1,000$ simulations). The figure also shows the bounds from the second-to-last iteration of the adaptive grid algorithm as a thick gray dashed line. These are almost identical to the actual state bounds in black, except for the capital lower bound. The average relative difference in the capital lower bounds between the final two algorithm iterations is less than $1 \%$.

The non-learning frameworks do not use the adaptive grid. This is for two reasons. First, the non-learning frameworks have low dimensionality and can be solved on a standard hyperrectangle grid. Second, without learning, the states often drift outside adapted approximation bounds when facing a misspecified damage function. If the policymaker's beliefs about the damage function are correct, the adaptive grid can 
Table A3: Weights on fitted spline bounds obtained from Step 3c in the adaptive grid algorithm. Weights on the previous iteration's bounds are 1 minus the weights in the table.

\begin{tabular}{lccccc}
\hline & $k$ & $E$ & $\mu$ & $\Sigma$ & $\mathcal{L}$ \\
\hline Upper Bound Weight & 0.3 & 0.4 & 0.5 & 0.0 & 0.0 \\
Lower Bound Weight & 0.1 & 0.3 & 0.5 & 0.0 & 0.5 \\
\hline
\end{tabular}

easily be used for a non-learning policymaker. The state bounds for the non-learning frameworks are contained in Table A2.

Error analysis I investigate the accuracy of the model by calculating the relative differences in simulated policy trajectories when (i) increasing the number of unique collocation points by 1 by over each dimension (e.g. capital goes from $4 \rightarrow 5$, cumulative emissions goes from $5 \rightarrow 6$, etc), (ii) increasing the number of quadrature points by 2 over the $d_{2}$ distribution and the joint $\left\{d_{1}, \omega_{t+1}\right\}$ distribution, (iii) increasing the collocation upper bound by $10 \%$ and decreasing the collocation lower bound by $10 \%$ for the non-adapted grids, ${ }^{8}$ and (iv) only doing the first 10 adaptive grid iterations instead of the full 20. For the frameworks with learning, (i)-(iii) use the same adaptive grid bounds as the baseline results. Tables A4 displays the maximum and average relative differences of the simulated trajectories' carbon taxes and consumption levels over the first 100 years.

Average differences in taxes and consumption for all frameworks are on the order of $10^{-3}$ or smaller, while the maximum error is larger but still relatively small. ${ }^{9}$

\section{B Carbon Tax Derivation}

The conventional definition of the time $t$ carbon tax used in the dynamic stochastic integrated assessment literature is that it is the shadow cost of time $t$ emissions. It is converted into dollar terms using time $t$ marginal utility evaluated at the optimal consumption level. Without loss of generality I derive the carbon tax using the value

\footnotetext{
${ }^{8}$ For fractional net output I reduce the lower bound by $20 \%$ since increasing the upper bound beyond 1.0 corresponds to generating more than $100 \%$ of possible output without climate change.

${ }^{9}$ Changing the number of collocation points on capital by 2 instead of 1 generates similarly sized errors of about $10^{-3}$ for averages and $10^{-2}$ for maximums.
} 
Table A4: Average and maximum differences in carbon taxes and consumption from increasing the number of collocation grid points or quadrature points, expanding the size of the approximation domain for the non-learning frameworks, and performing 10 fewer iterations of the adaptive grid algorithm for the frameworks with learning. The average and maximum carbon tax trajectory differences are taken from 50,000 simulations over 2005-2105 where each simulation has a random draw of $\left\{d_{1}, d_{2}, \omega_{2005}, \ldots, \omega_{2105}\right\}$.

\begin{tabular}{cccccc}
\hline Parameters Changed & Relative Differences & Uncertainty & Learning & RC & RC+L \\
\hline \# Collocation Points: & Tax: Max & $1.1 \%$ & $1.4 \%$ & $1.3 \%$ & $0.7 \%$ \\
Add 1 unique point & Tax: Avg & $0.2 \%$ & $0.5 \%$ & $0.2 \%$ & $0.4 \%$ \\
to each state & Consumption: Max & $0.2 \%$ & $0.08 \%$ & $0.2 \%$ & $0.06 \%$ \\
& Consumption: Avg & $0.02 \%$ & $9 \times 10^{-3} \%$ & $0.02 \%$ & $8 \times 10^{-3} \%$ \\
\hline \# Quadrature Points: & Tax: Max & $0.06 \%$ & $0.6 \%$ & $0.05 \%$ & $0.5 \%$ \\
Add 2 unique points & Tax: Avg & $0.02 \%$ & $0.3 \%$ & $0.02 \%$ & $0.2 \%$ \\
to both distributions: & Consumption: Max & $0.03 \%$ & $0.03 \%$ & $0.03 \%$ & $0.03 \%$ \\
$\left\{d_{2}\right\}$ and $\left\{d_{1}, \omega_{t+1}\right\}$ & Consumption: Avg & $7 \times 10^{-3} \%$ & $8 \times 10^{-3} \%$ & $7 \times 10^{-3} \%$ & $8 \times 10^{-3} \%$ \\
\hline Domain Bounds: & Tax: Max & $0.8 \%$ & - & $0.9 \%$ & - \\
Increase UB and & Tax: Avg & $0.3 \%$ & - & $0.3 \%$ & - \\
decrease LB by 10\% & Consumption: Max & $0.2 \%$ & - & $0.2 \%$ & - \\
& Consumption: Avg & $0.09 \%$ & - & $0.09 \%$ & - \\
\hline \multirow{2}{*}{ Adaptive Iterations: } & Tax: Max & - & $0.8 \%$ & - & $0.8 \%$ \\
Decrease from 20 to 10 & Tax: Avg & - & $0.3 \%$ & - & $0.3 \%$ \\
& Consumption: Max & - & $0.2 \%$ & - & $0.2 \%$ \\
& Consumption: Avg & - & $0.06 \%$ & - & $0.06 \%$ \\
\hline
\end{tabular}


function for the robust control and learning framework. Recall the right hand side of the Bellman equation is

$$
V_{t}\left(\boldsymbol{S}_{t}\right)=\max _{c_{t}, \alpha_{t}}\left\{U\left(c_{t}\right)+\mathbb{E}_{d_{2}}\left[-\theta \log \left(\mathbb{E}_{d_{1}, \omega_{t+1}}\left[\exp \left(\frac{-\beta_{t} V_{t+1}\left(\boldsymbol{S}_{\boldsymbol{t}+\mathbf{1}}\right)}{\theta}\right)\right]\right)\right]\right\}
$$

where $\mathbf{S}_{\mathbf{t}+\mathbf{1}} \equiv\left\{k_{t+1}, E_{t+1}, \mathcal{L}_{t+1}, \mu_{t+1}, \Sigma_{t+1}\right\}$ and expectations are taken using the time $t$ information set. To economize on space I omit the value function arguments for now. The shadow cost of emissions is the negative partial derivative of the right hand side of the Bellman with respect to time t emissions $e_{t}$, which yields

$$
\theta \mathbb{E}_{d_{2}}\left\{\frac{\mathbb{E}_{d_{1}, \omega_{t+1}}\left[-\frac{\beta_{t}}{\theta} \exp \left(\frac{-\beta_{t} V_{t+1}}{\theta}\right) \frac{\partial V_{t+1}}{\partial \mathbf{S}_{\mathbf{t}+1}} \frac{\partial \mathbf{S}_{\mathbf{t}+1}}{\partial e_{t}}\right]}{\mathbb{E}_{d_{1}, \omega_{t+1}}\left[\exp \left(\frac{-\beta_{t} V_{t+1}}{\theta}\right)\right]}\right\}
$$

The $\theta$ terms cancel. Expand the expectation term over $d_{1}, \omega_{t+1}$ in the numerator of the fraction using the covariance identity to obtain$$
\beta_{t} \mathbb{E}_{d_{2}}\left\{\frac{\mathbb{E}_{d_{1}, \omega_{t+1}}\left[\exp \left(\frac{-\beta_{t} V_{t+1}}{\theta}\right)\right] \mathbb{E}_{d_{1}, \omega_{t+1}}\left[\frac{-\partial V_{t+1}}{\partial \mathbf{S}_{\mathbf{t}+1}} \frac{\partial \mathbf{S}_{t+1}}{\partial e_{t}}\right]}{\mathbb{E}_{d_{1}, \omega_{t+1}}\left[\exp \left(\frac{-\beta_{t} V_{t+1}}{\theta}\right)\right]}+\frac{\operatorname{cov}_{d_{1}, \omega_{t+1}}\left(\exp \left(\frac{-\beta_{t} V_{t+1}}{\theta}\right), \frac{-\partial V_{t+1}}{\partial \mathbf{S}_{\mathbf{t}+1}} \frac{\partial \mathbf{S}_{\mathbf{t}+1}}{\partial e_{t}}\right)}{\mathbb{E}_{d_{1}, \omega_{t+1}}\left[\exp \left(\frac{-\beta_{t} V_{t+1}}{\theta}\right)\right]}\right\} .
$$

We can cancel the expectations over the exponentials in the first term, and bring the denominator of the second term inside of the covariance operator such that it multiplies the first argument. The last step to obtain the optimal carbon tax is to divide by $u^{\prime}\left(c_{t}\right)$ to translate into dollar terms

$$
\frac{\beta_{t}}{u^{\prime}\left(c_{t}\right)} \mathbb{E}_{d_{2}}\left\{\mathbb{E}_{d_{1}, \omega_{t+1}}\left[\frac{-\partial V_{t+1}}{\partial \mathbf{S}_{\mathbf{t}+\mathbf{1}}} \frac{\partial \mathbf{S}_{\mathbf{t}+\mathbf{1}}}{\partial e_{t}}\right]+\operatorname{cov}_{d_{1}, \omega_{t+1}}\left(\frac{\exp \left(\frac{-\beta_{t} V_{t+1}}{\theta}\right)}{\mathbb{E}_{d_{1}, \omega_{t+1}}\left[\exp \left(\frac{-\beta_{t} V_{t+1}}{\theta}\right)\right]}, \frac{-\partial V_{t+1}}{\partial \mathbf{S}_{\mathbf{t + 1}}} \frac{\partial \mathbf{S}_{\mathbf{t}+\mathbf{1}}}{\partial e_{t}}\right)\right\} .
$$

The first term is the standard carbon tax expression without robust control. The second term is the additively separable adjustment for robust control.

Now focus on the first term (the standard carbon tax expression term) and ignore the leading $\frac{\beta_{t}}{u^{\prime}\left(c_{t}\right)}$ for clarity. Expanding out the state vector, and recognizing that 
time $t+1$ capital is not a function of time $t$ emissions, we have that

$$
\begin{aligned}
\mathbb{E}_{d_{2}, d_{1}, \omega_{t+1}}\left[\frac{-\partial V_{t+1}}{\partial \mathbf{S}_{\mathbf{t}+\mathbf{1}}} \frac{\partial \mathbf{S}_{\mathbf{t}+\mathbf{1}}}{\partial e_{t}}\right] & =\mathbb{E}_{d_{2}, d_{1}, \omega_{t+1}}\left[\frac{-\partial V_{t+1}}{\partial E_{t+1}} \frac{\partial E_{t+1}}{\partial e_{t}}\right]+\mathbb{E}_{d_{2}, d_{1}, \omega_{t+1}}\left[\frac{-\partial V_{t+1}}{\partial \mathcal{L}_{t+1}} \frac{\partial \mathcal{L}_{t}}{\partial e_{t}}\right] \\
& +\mathbb{E}_{d_{2}, d_{1}, \omega_{t+1}}\left[\frac{-\partial V_{t+1}}{\partial \mu_{t+1}} \frac{\partial \mu_{t+1}}{\partial e_{t}}\right]+\mathbb{E}_{d_{2}, d_{1}, \omega_{t+1}}\left[\frac{-\partial V_{t+1}}{\partial \Sigma_{t+1}} \frac{\partial \Sigma_{t+1}}{\partial e_{t}}\right]
\end{aligned}
$$

Next pass the expectations through. Recognize $\frac{\partial \mathcal{L}_{t+1}}{\partial e_{t}}$ and $\frac{\partial \mu_{t+1}}{\partial e_{t}}$ are random variables so we can apply the covariance identity to the expectations over those terms. And finally note that $\mathbb{E}_{d_{2}, d_{1}, \omega_{t+1}}\left[\frac{\partial \mu_{t+1}}{\partial e_{t}}\right]=0$ for a Bayesian, and $\frac{\partial E_{t+1}}{\partial e_{t}}=1$ by definition. Then we arrive at the final standard carbon tax expression before performing Taylor expansions

$$
\begin{aligned}
\mathbb{E}_{d_{2}, d_{1}, \omega_{t+1}}\left[\frac{-\partial V_{t+1}}{\partial \mathbf{S}_{\mathbf{t}+\mathbf{1}}} \frac{\partial \mathbf{S}_{\mathbf{t}+\mathbf{1}}}{\partial e_{t}}\right] & =\mathbb{E}_{d_{2}, d_{1}, \omega_{t+1}}\left[\frac{-\partial V_{t+1}}{\partial E_{t+1}}\right] \\
& +\mathbb{E}_{d_{2}, d_{1}, \omega_{t+1}}\left[\frac{-\partial V_{t+1}}{\partial \mathcal{L}_{t+1}}\right] \mathbb{E}_{d_{2}, d_{1}, \omega_{t+1}}\left[\frac{\partial \mathcal{L}_{t}}{\partial e_{t}}\right]+\operatorname{cov}_{d_{2}, d_{1}, \omega_{t+1}}\left(\frac{-\partial V_{t+1}}{\partial \mathcal{L}_{t+1}}, \frac{\partial \mathcal{L}_{t+1}}{\partial e_{t}}\right) \\
& +\operatorname{cov}_{d_{2}, d_{1}, \omega_{t+1}}\left(\frac{-\partial V_{t+1}}{\partial \mu_{t+1}}, \frac{\partial \mu_{t+1}}{\partial e_{t}}\right)+\mathbb{E}_{d_{2}, d_{1}, \omega_{t+1}}\left[\frac{-\partial V_{t+1}}{\partial \Sigma_{t+1}}\right] \frac{\partial \Sigma_{t+1}}{\partial e_{t}}
\end{aligned}
$$

Recall that for a simple bivariate case, a second-order Taylor expansion of $E_{x, y}[f(x, y)]$ about $(c, d)$ is

$$
\begin{aligned}
E_{x, y}[f(x, y)] \approx & E_{x, y}[f(c, d) \\
& +f_{x}(x, y)(x-c)+f_{y}(c, d)(y-d) \\
& +\frac{1}{2}\left[f_{x y}(c, d)(x-c)^{2}+f_{x y}(c, d)(x-c)(y-d)\right. \\
& \left.\left.+f_{y x}(c, d)(y-d)(x-c)+f_{y y}(c, d)(y-d)^{2}\right]\right]
\end{aligned}
$$

where the term on the first line and on right hand side of the equality is the zerothorder term, the second line contains the first-order terms, and the third and fourth lines contain the second-order terms. Now perform a second-order Taylor expansion of the expectations of the value function partial derivatives around

$$
v:=\left\{k_{t+1}, E_{t+1}, \mathbb{E}_{d_{2}, d_{1}, \omega_{t+1}}\left[\mathcal{L}_{t+1}\right], \mathbb{E}_{d_{2}, d_{1}, \omega_{t+1}}\left[\mu_{t+1}\right], \Sigma_{t+1}\right\}
$$


where $\mathbb{E}_{d_{2}, d_{1}, \omega_{t+1}}\left[\mu_{t+1}\right]=\mu_{t}$.

Here I show the second-order Taylor expansion for $\mathbb{E}_{d_{2}, d_{1}, \omega_{t+1}}\left[\frac{-\partial V_{t+1}}{\partial E_{t+1}}\right]$ evaluated at $v$. It consists of 1 zeroth-order Taylor term, five first-order Taylor terms, and twenty five second-order Taylor terms

$$
\begin{aligned}
& \mathbb{E}_{d_{2}, d_{1}, \omega_{t+1}}\left[\frac{-\partial V_{t+1}}{\partial E_{t+1}}\right] \approx \mathbb{E}_{d_{2}, d_{1}, \omega_{t+1}}\left[\left.\frac{-\partial V_{t+1}}{\partial E_{t+1}}\right|_{v}\right. \\
& +\left.\frac{-\partial^{2} V_{t+1}}{\partial E_{t+1} \partial k_{t+1}}\right|_{v}\left(k_{t+1}-\mathbb{E}_{d_{2}, d_{1}, \omega_{t+1}}\left[k_{t+1}\right]\right) \\
& +\left.\frac{-\partial^{2} V_{t+1}}{\partial E_{t+1}^{2}}\right|_{v}\left(E_{t+1}-\mathbb{E}_{d_{2}, d_{1}, \omega_{t+1}}\left[E_{t+1}\right]\right) \\
& +\left.\frac{-\partial^{2} V_{t+1}}{\partial E_{t+1} \partial \mathcal{L}_{t+1}}\right|_{v}\left(\mathcal{L}_{t+1}-\mathbb{E}_{d_{2}, d_{1}, \omega_{t+1}}\left[\mathcal{L}_{t+1}\right]\right) \\
& +\left.\frac{-\partial^{2} V_{t+1}}{\partial E_{t+1} \partial \mu_{t+1}}\right|_{v}\left(\mu_{t+1}-\mathbb{E}_{d_{2}, d_{1}, \omega_{t+1}}\left[\mu_{t+1}\right]\right) \\
& +\left.\frac{-\partial^{2} V_{t+1}}{\partial E_{t+1} \partial \Sigma_{t+1}}\right|_{v}\left(\Sigma_{t+1}-\mathbb{E}_{d_{2}, d_{1}, \omega_{t+1}}\left[\Sigma_{t+1}\right]\right) \\
& +\left.\frac{1}{2} \frac{-\partial^{3} V_{t+1}}{\partial E_{t+1} \partial k_{t+1}^{2}}\right|_{v}\left(k_{t+1}-\mathbb{E}_{d_{2}, d_{1}, \omega_{t+1}}\left[k_{t+1}\right]\right)^{2} \quad \text { (Second-order) } \\
& +\left.\frac{1}{2} \frac{-\partial^{3} V_{t+1}}{\partial E_{t+1} \partial k_{t+1} \partial E_{t+1}}\right|_{v}\left(k_{t+1}-\mathbb{E}_{d_{2}, d_{1}, \omega_{t+1}}\left[k_{t+1}\right]\right)\left(E_{t+1}-\mathbb{E}_{d_{2}, d_{1}, \omega_{t+1}}\left[E_{t+1}\right]\right) \\
& +\left.\frac{1}{2} \frac{-\partial^{3} V_{t+1}}{\partial E_{t+1} \partial k_{t+1} \partial \mathcal{L}_{t+1}}\right|_{v}\left(k_{t+1}-\mathbb{E}_{d_{2}, d_{1}, \omega_{t+1}}\left[k_{t+1}\right]\right)\left(\mathcal{L}_{t+1}-\mathbb{E}_{d_{2}, d_{1}, \omega_{t+1}}\left[\mathcal{L}_{t+1}\right]\right) \\
& +\left.\frac{1}{2} \frac{-\partial^{3} V_{t+1}}{\partial E_{t+1} \partial k_{t+1} \partial \mu_{t+1}}\right|_{v}\left(k_{t+1}-\mathbb{E}_{d_{2}, d_{1}, \omega_{t+1}}\left[k_{t+1}\right]\right)\left(\mu_{t+1}-\mathbb{E}_{d_{2}, d_{1}, \omega_{t+1}}\left[\mu_{t+1}\right]\right) \\
& +\left.\frac{1}{2} \frac{-\partial^{3} V_{t+1}}{\partial E_{t+1} \partial k_{t+1} \partial \Sigma_{t+1}}\right|_{v}\left(k_{t+1}-\mathbb{E}_{d_{2}, d_{1}, \omega_{t+1}}\left[k_{t+1}\right]\right)\left(\Sigma_{t+1}-\mathbb{E}_{d_{2}, d_{1}, \omega_{t+1}}\left[\Sigma_{t+1}\right]\right) \\
& +\left.\frac{1}{2} \frac{-\partial^{3} V_{t+1}}{\partial E_{t+1}^{2} \partial k_{t+1}}\right|_{v}\left(E_{t+1}-\mathbb{E}_{d_{2}, d_{1}, \omega_{t+1}}\left[E_{t+1}\right]\right)\left(k_{t+1}-\mathbb{E}_{d_{2}, d_{1}, \omega_{t+1}}\left[k_{t+1}\right]\right) \\
& +\left.\frac{1}{2} \frac{-\partial^{3} V_{t+1}}{\partial E_{t+1}^{3}}\right|_{v}\left(E_{t+1}-\mathbb{E}_{d_{2}, d_{1}, \omega_{t+1}}\left[E_{t+1}\right]\right)^{2} \\
& +\left.\frac{1}{2} \frac{-\partial^{3} V_{t+1}}{\partial E_{t+1}^{2} \partial \mathcal{L}_{t+1}}\right|_{v}\left(E_{t+1}-\mathbb{E}_{d_{2}, d_{1}, \omega_{t+1}}\left[E_{t+1}\right]\right)\left(\mathcal{L}_{t+1}-\mathbb{E}_{d_{2}, d_{1}, \omega_{t+1}}\left[\mathcal{L}_{t+1}\right]\right) \\
& +\left.\frac{1}{2} \frac{-\partial^{3} V_{t+1}}{\partial E_{t+1}^{2} \partial \mu_{t+1}}\right|_{v}\left(E_{t+1}-\mathbb{E}_{d_{2}, d_{1}, \omega_{t+1}}\left[E_{t+1}\right]\right)\left(\mu_{t+1}-\mathbb{E}_{d_{2}, d_{1}, \omega_{t+1}}\left[\mu_{t+1}\right]\right)
\end{aligned}
$$




$$
\begin{aligned}
& +\left.\frac{1}{2} \frac{-\partial^{3} V_{t+1}}{\partial E_{t+1}^{2} \partial \Sigma_{t+1}}\right|_{v}\left(E_{t+1}-\mathbb{E}_{d_{2}, d_{1}, \omega_{t+1}}\left[E_{t+1}\right]\right)\left(\Sigma_{t+1}-\mathbb{E}_{d_{2}, d_{1}, \omega_{t+1}}\left[\Sigma_{t+1}\right]\right) \\
& +\left.\frac{1}{2} \frac{-\partial^{3} V_{t+1}}{\partial E_{t+1} \partial \mathcal{L}_{t+1} \partial k_{t+1}}\right|_{v}\left(\mathcal{L}_{t+1}-\mathbb{E}_{d_{2}, d_{1}, \omega_{t+1}}\left[\mathcal{L}_{t+1}\right]\right)\left(k_{t+1}-\mathbb{E}_{d_{2}, d_{1}, \omega_{t+1}}\left[k_{t+1}\right]\right) \\
& +\left.\frac{1}{2} \frac{-\partial^{3} V_{t+1}}{\partial E_{t+1} \partial \mathcal{L}_{t+1} \partial E_{t+1}}\right|_{v}\left(\mathcal{L}_{t+1}-\mathbb{E}_{d_{2}, d_{1}, \omega_{t+1}}\left[\mathcal{L}_{t+1}\right]\right)\left(E_{t+1}-\mathbb{E}_{d_{2}, d_{1}, \omega_{t+1}}\left[E_{t+1}\right]\right) \\
& +\left.\frac{1}{2} \frac{-\partial^{3} V_{t+1}}{\partial E_{t+1} \partial \mathcal{L}_{t+1}^{2}}\right|_{v}\left(\mathcal{L}_{t+1}-\mathbb{E}_{d_{2}, d_{1}, \omega_{t+1}}\left[\mathcal{L}_{t+1}\right]\right)^{2} \\
& +\left.\frac{1}{2} \frac{-\partial^{3} V_{t+1}}{\partial E_{t+1} \partial \mathcal{L}_{t+1} \partial \mu_{t+1}}\right|_{v}\left(\mathcal{L}_{t+1}-\mathbb{E}_{d_{2}, d_{1}, \omega_{t+1}}\left[\mathcal{L}_{t+1}\right]\right)\left(\mu_{t+1}-\mathbb{E}_{d_{2}, d_{1}, \omega_{t+1}}\left[\mu_{t+1}\right]\right) \\
& +\left.\frac{1}{2} \frac{-\partial^{3} V_{t+1}}{\partial E_{t+1} \partial \mathcal{L}_{t+1} \partial \Sigma_{t+1}}\right|_{v}\left(\mathcal{L}_{t+1}-\mathbb{E}_{d_{2}, d_{1}, \omega_{t+1}}\left[\mathcal{L}_{t+1}\right]\right)\left(\Sigma_{t+1}-\mathbb{E}_{d_{2}, d_{1}, \omega_{t+1}}\left[\Sigma_{t+1}\right]\right) \\
& +\left.\frac{1}{2} \frac{-\partial^{3} V_{t+1}}{\partial E_{t+1} \partial \mu_{t+1} \partial k_{t+1}}\right|_{v}\left(\mu_{t+1}-\mathbb{E}_{d_{2}, d_{1}, \omega_{t+1}}\left[\mu_{t+1}\right]\right)\left(k_{t+1}-\mathbb{E}_{d_{2}, d_{1}, \omega_{t+1}}\left[k_{t+1}\right]\right) \\
& +\left.\frac{1}{2} \frac{-\partial^{3} V_{t+1}}{\partial E_{t+1} \partial \mu_{t+1} \partial E_{t+1}}\right|_{v}\left(\mu_{t+1}-\mathbb{E}_{d_{2}, d_{1}, \omega_{t+1}}\left[\mu_{t+1}\right]\right)\left(E_{t+1}-\mathbb{E}_{d_{2}, d_{1}, \omega_{t+1}}\left[E_{t+1}\right]\right) \\
& +\left.\frac{1}{2} \frac{-\partial^{3} V_{t+1}}{\partial E_{t+1} \partial \mu_{t+1} \partial \mathcal{L}_{t+1}}\right|_{v}\left(\mu_{t+1}-\mathbb{E}_{d_{2}, d_{1}, \omega_{t+1}}\left[\mu_{t+1}\right]\right)\left(\mathcal{L}_{t+1}-\mathbb{E}_{d_{2}, d_{1}, \omega_{t+1}}\left[\mathcal{L}_{t+1}\right]\right) \\
& +\left.\frac{1}{2} \frac{-\partial^{3} V_{t+1}}{\partial E_{t+1} \partial \mu_{t+1}^{2}}\right|_{v}\left(\mu_{t+1}-\mathbb{E}_{d_{2}, d_{1}, \omega_{t+1}}\left[\mu_{t+1}\right]\right)^{2} \\
& +\left.\frac{1}{2} \frac{-\partial^{3} V_{t+1}}{\partial E_{t+1} \partial \mu_{t+1} \partial \Sigma_{t+1}}\right|_{v}\left(\mu_{t+1}-\mathbb{E}_{d_{2}, d_{1}, \omega_{t+1}}\left[\mu_{t+1}\right]\right)\left(\Sigma_{t+1}-\mathbb{E}_{d_{2}, d_{1}, \omega_{t+1}}\left[\Sigma_{t+1}\right]\right) \\
& +\left.\frac{1}{2} \frac{-\partial^{3} V_{t+1}}{\partial E_{t+1} \partial \Sigma_{t+1} \partial k_{t+1}}\right|_{v}\left(\Sigma_{t+1}-\mathbb{E}_{d_{2}, d_{1}, \omega_{t+1}}\left[\Sigma_{t+1}\right]\right)\left(k_{t+1}-\mathbb{E}_{d_{2}, d_{1}, \omega_{t+1}}\left[k_{t+1}\right]\right) \\
& +\left.\frac{1}{2} \frac{-\partial^{3} V_{t+1}}{\partial E_{t+1} \partial \Sigma_{t+1} \partial E_{t+1}}\right|_{v}\left(\Sigma_{t+1}-\mathbb{E}_{d_{2}, d_{1}, \omega_{t+1}}\left[\Sigma_{t+1}\right]\right)\left(E_{t+1}-\mathbb{E}_{d_{2}, d_{1}, \omega_{t+1}}\left[E_{t+1}\right]\right) \\
& +\left.\frac{1}{2} \frac{-\partial^{3} V_{t+1}}{\partial E_{t+1} \partial \Sigma_{t+1} \partial \mathcal{L}_{t+1}}\right|_{v}\left(\Sigma_{t+1}-\mathbb{E}_{d_{2}, d_{1}, \omega_{t+1}}\left[\Sigma_{t+1}\right]\right)\left(\mathcal{L}_{t+1}-\mathbb{E}_{d_{2}, d_{1}, \omega_{t+1}}\left[\mathcal{L}_{t+1}\right]\right) \\
& +\left.\frac{1}{2} \frac{-\partial^{3} V_{t+1}}{\partial E_{t+1} \partial \Sigma_{t+1} \partial \mu_{t+1}}\right|_{v}\left(\Sigma_{t+1}-\mathbb{E}_{d_{2}, d_{1}, \omega_{t+1}}\left[\Sigma_{t+1}\right]\right)\left(\mu_{t+1}-\mathbb{E}_{d_{2}, d_{1}, \omega_{t+1}}\left[\mu_{t+1}\right]\right) \\
& \left.+\left.\frac{1}{2} \frac{-\partial^{3} V_{t+1}}{\partial E_{t+1} \partial \Sigma_{t+1}^{2}}\right|_{v}\left(\Sigma_{t+1}-\mathbb{E}_{d_{2}, d_{1}, \omega_{t+1}}\left[\Sigma_{t+1}\right]\right)^{2}\right] \text {. }
\end{aligned}
$$

Note that the expectation is taken over all terms in the expansion. The term on the right hand side of the equality on the first line is the zeroth-order term, the value 
function partial evaluated at $v$, which is the state vector about which I do the Taylor expansion. This term is not random since it is evaluated at a specific state, so the expectation passes through.

The next five lines are the first-order Taylor terms. Here, another partial derivative of the value function is taken with respect to each state, then it is multiplied by the difference between the state and that state's value at $v$, which is just the expectation of the state. The value function terms here have two partial derivatives, and are evaluated at $v$. They also are not random so the expectation passes through those terms onto the terms capturing the difference between the state and its value at $v$. The expectation of the difference between a variable and its expectation is zero so all first-order Taylor terms of the expansion are zero.

The second-order Taylor terms take another partial derivative, so these are the terms that include all of the triple value function partial derivatives. The triple partials are multiplied by the product of two differences between a state and that state's value at $v$. The state in each difference corresponds to the state with respect to which the second and third partial derivatives are taken. As in zeroth-order and firstorder Taylor terms, the triple partial derivatives are not random and the expectation passes through all of them onto the two difference terms. In the cases where at least one of the differences in the second-order Taylor term consists of a deterministic state $\left(k_{t+1}, E_{t+1}, \Sigma_{t+1}\right)$, the expectation of the product of the two differences is again zero and the Taylor terms are zero. The remaining case is when both states in the product of differences are uncertain $\left(\mathcal{L}_{t+1}, \mu_{t+1}\right)$. After passing the expectation through the triple partial derivative terms, the difference terms are variances (e.g. $\mathbb{E}_{d_{2}, d_{1}, \omega_{t+1}}\left[\left(\mu_{t+1}-\mathbb{E}_{d_{2}, d_{1}, \omega_{t+1}}\left[\mu_{t+1}\right]\right)^{2}\right]=\operatorname{var}_{d_{2}, d_{1}, \omega_{t+1}}\left(\mu_{t+1}\right)$ on the seventh to last line) or covariances (e.g. $\mathbb{E}_{d_{2}, d_{1}, \omega_{t+1}}\left[\left(\mu_{t+1}-\mathbb{E}_{d_{2}, d_{1}, \omega_{t+1}}\left[\mu_{t+1}\right]\right)\left(\mathcal{L}_{t+1}-\mathbb{E}_{d_{2}, d_{1}, \omega_{t+1}}\left[\mathcal{L}_{t+1}\right]\right)\right]=$ $\operatorname{cov}_{d_{2}, d_{1}, \omega_{t+1}}\left(\mu_{t+1}, \mathcal{L}_{t+1}\right)$ on the eighth to last line). Similar logic follows for Taylor expansions for the other value function partials in equation (2).

Now define

$$
c e:=\left\{k_{t+1}, E_{t+1},\left.\mathcal{L}_{t+1}\right|_{d_{1}=\exp \left(\mu_{c}+\frac{1}{2} \sigma_{c}^{2}\right), \omega_{t+1}=\exp \left(\mu_{\omega}+\frac{1}{2} \sigma_{\omega}^{2}\right)}, \mu_{t}, 0\right\} .
$$

$c e$ is a certainty state. Terms evaluated at ce are the terms that would arise if the policymaker happened to arrive at the same state at time $t$ but states transitioned deterministically (with $d_{1}, d_{2}, \omega_{t}$ fixed to their expectations). I will add and sub- 
tract value function partial derivatives evaluated at ce, effectively adding 0 , to obtain the certainty tax and the state uncertainty adjustment. After omitting the Taylor terms that are zero, the expectation of the cumulative emissions partial derivative is approximately

$$
\begin{aligned}
\mathbb{E}_{d_{2}, d_{1}, \omega_{t+1}}\left[\frac{-\partial V_{t+1}}{\partial E_{t+1}}\right] & \left.\approx \frac{-\partial V_{t+1}}{\partial E_{t+1}}\right|_{c e}+\left[\left.\frac{-\partial V_{t+1}}{\partial E_{t+1}}\right|_{v}-\left.\frac{-\partial V_{t+1}}{\partial E_{t+1}}\right|_{c e}\right] \\
+ & \left.\frac{1}{2} \frac{-\partial^{3} V_{t+1}}{\partial E_{t+1} \partial \mathcal{L}_{t+1}^{2}}\right|_{v} \operatorname{var}_{d_{2}, d_{1}, \omega_{t+1}}\left(\mathcal{L}_{t+1}\right)+\left.\frac{-\partial^{3} V_{t+1}}{\partial E_{t+1} \partial \mathcal{L}_{t+1} \partial \mu_{t+1}}\right|_{v} \operatorname{cov}_{d_{2}, d_{1}, \omega_{t+1}}\left(\mathcal{L}_{t+1}, \mu_{t+1}\right) \\
& +\left.\frac{1}{2} \frac{-\partial^{3} V_{t+1}}{\partial E_{t+1} \partial \mu_{t+1}^{2}}\right|_{v} \operatorname{var}_{d_{2}, d_{1}, \omega_{t+1}}\left(\mu_{t+1}\right)
\end{aligned}
$$

The expectation of the fractional net output partial derivative is approximately

$$
\begin{aligned}
\mathbb{E}_{d_{2}, d_{1}, \omega_{t+1}}\left[\frac{-\partial V_{t+1}}{\partial \mathcal{L}_{t+1}}\right] & \left.\approx \frac{-\partial V_{t+1}}{\partial \mathcal{L}_{t+1}}\right|_{c e}+\left[\left.\frac{-\partial V_{t+1}}{\partial \mathcal{L}_{t+1}}\right|_{v}-\left.\frac{-\partial V_{t+1}}{\partial \mathcal{L}_{t+1}}\right|_{c e}\right] \\
& +\left.\frac{1}{2} \frac{-\partial^{3} V_{t+1}}{\partial \mathcal{L}_{t+1}^{3}}\right|_{v} \operatorname{var}_{d_{2}, d_{1}, \omega_{t+1}}\left(\mathcal{L}_{t+1}\right)+\left.\frac{-\partial^{3} V_{t+1}}{\partial \mathcal{L}_{t+1}^{2} \partial \mu_{t+1}}\right|_{v} \operatorname{cov}_{d_{2}, d_{1}, \omega_{t+1}}\left(\mathcal{L}_{t+1}, \mu_{t+1}\right) \\
& +\left.\frac{1}{2} \frac{-\partial^{3} V_{t+1}}{\partial \mathcal{L}_{t+1} \partial \mu_{t+1}^{2}}\right|_{v} \operatorname{var}_{d_{2}, d_{1}, \omega_{t+1}}\left(\mu_{t+1}\right)
\end{aligned}
$$

The expectation of the scale parameter partial derivative is approximately

$$
\begin{aligned}
\mathbb{E}_{d_{2}, d_{1}, \omega_{t+1}}\left[\frac{-\partial V_{t+1}}{\partial \Sigma_{t+1}}\right] & \left.\approx \frac{-\partial V_{t+1}}{\partial \Sigma_{t+1}}\right|_{c e}+\left[\left.\frac{-\partial V_{t+1}}{\partial \Sigma_{t+1}}\right|_{v}-\left.\frac{-\partial V_{t+1}}{\partial \Sigma_{t+1}}\right|_{c e}\right] \\
& +\left.\frac{1}{2} \frac{-\partial^{3} V_{t+1}}{\partial \Sigma_{t+1} \partial \mathcal{L}_{t+1}^{2}}\right|_{v} \operatorname{var}_{d_{2}, d_{1}, \omega_{t+1}}\left(\mathcal{L}_{t+1}\right)+\left.\frac{-\partial^{3} V_{t+1}}{\partial \Sigma_{t+1} \partial \mathcal{L}_{t+1} \partial \mu_{t+1}}\right|_{v} \operatorname{cov}_{d_{2}, d_{1}, \omega_{t+1}}\left(\mathcal{L}_{t+1}, \mu_{t+1}\right) \\
& +\left.\frac{1}{2} \frac{-\partial^{3} V_{t+1}}{\partial \Sigma_{t+1} \partial \mu_{t+1}^{2}}\right|_{v} \operatorname{var}_{d_{2}, d_{1}, \omega_{t+1}}\left(\mu_{t+1}\right)
\end{aligned}
$$

Substituting the Taylor expansion terms into equation (2) and then substituting equation (2) into equation (1) yields the full carbon tax expression.

\section{Certainty Tax}

Figure A3 displays the certainty tax channel for each framework. The certainty tax is the remaining difference between the carbon tax in Figure 5 and the uncertainty channels in Figure 7 in the main text. 


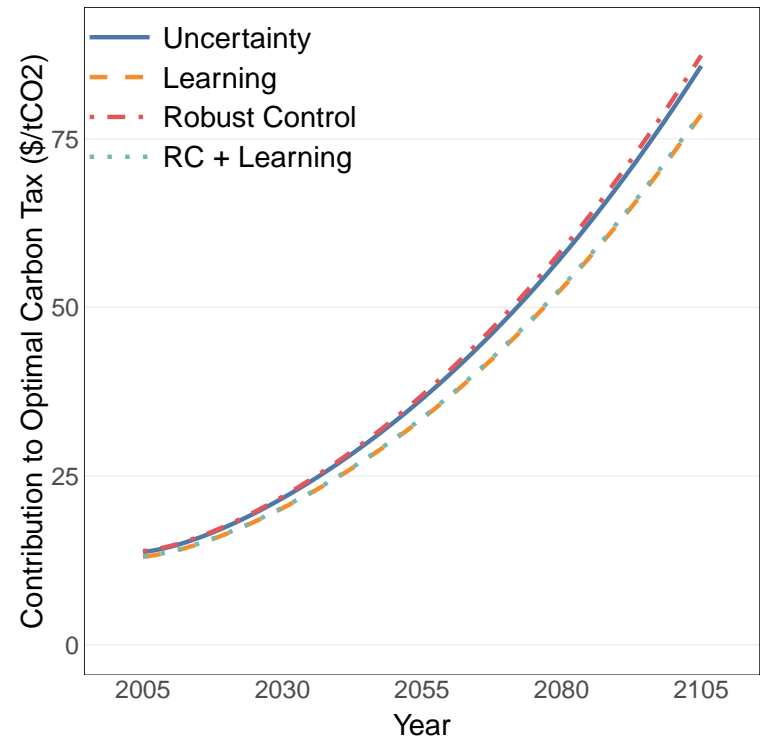

Figure A3: The mean certainty tax channel over 50,000 simulations for each framework. Each simulation samples a different set of $\left\{d_{1}, d_{2}, \omega_{2005}, \ldots, \omega_{2105}\right\}$.

\section{References}

Allen, Myles R, David J Frame, Chris Huntingford, Chris D Jones, Jason A Lowe, Malte Meinshausen, and Nicolai Meinshausen (2009) "Warming caused by cumulative carbon emissions towards the trillionth tonne," Nature, Vol. 458, No. 7242, pp. $1163-1166$.

Berger, Loic and Massimo Marinacci (2017) "Model Uncertainty in Climate Change Economics," No. Cmcc, pp. 1-33.

Cai, Yongyang (2018) "Computational Methods in Environmental and Resource Economics," Annual Review of Resource Economics, Vol. 11.

Cai, Yongyang, Kenneth L Judd, Timothy M Lenton, Thomas S Lontzek, and Daiju Narita (2015) "Environmental tipping points significantly affect the cost- benefit assessment of climate policies," Proceedings of the National Academy of Sciences, p. 201503890.

Cai, Yongyang, Kenneth L. Judd, and Thomas S. Lontzek (Forthcoming) "The Social Cost of Carbon with Economic and Climate Risks," Journal of Political Economy.

Cai, Yongyang, Timothy M Lenton, and Thomas S Lontzek (2016) "Risk of multiple interacting tipping points should encourage rapid CO 2 emission reduction," Nature Climate Change, Vol. 6, No. 5, p. 520. 
Dietz, Simon and Frank Venmans (2019) "Cumulative carbon emissions and economic policy: in search of general principles," Journal of Environmental Economics and Management, Vol. 96, pp. 108-129.

IPCC (2013) "Climate Change 2013: The Physical Science Basis. Contribution of Working Group I to the Fifth Assessment Report of the Intergovernmental Panel on Climate Change," Cambridge University Press, Cambridge, United Kingdom and New York, NY, USA.

(2014) Climate Change 2013: The Physical Science Basis: Working Group I Contribution to the Fifth Assessment Report of the Intergovernmental Panel on Climate Change: Cambridge University Press.

Knutti, Reto, Maria A. A. Rugenstein, and Gabriele C. Hegerl (2017) "Beyond equilibrium climate sensitivity," Nature Geoscience, Vol. 10, No. 10, p. 727.

Leduc, Martin, H Damon Matthews, and Ramón de Elía (2015) "Quantifying the limits of a linear temperature response to cumulative CO2 emissions," Journal of Climate, Vol. 28, No. 24, pp. 9955-9968.

Lontzek, Thomas S., Yongyang Cai, Kenneth L. Judd, and Timothy M. Lenton (2015) "Stochastic integrated assessment of climate tipping points indicates the need for strict climate policy," Nature Climate Change, Vol. 5, No. 5, pp. 441-444.

MacDougall, Andrew H and Pierre Friedlingstein (2015) "The Origin and Limits of the Near Proportionality Between Climate Warming and Cumulative CO2 Emissions," Journal of Climate, Vol. 28, No. 10, pp. 4217-4230.

Matthews, H Damon and Ken Caldeira (2008) "Stabilizing Climate Requires NearZero Emissions," Geophysical Research Letters, Vol. 35, No. 4.

Matthews, H Damon, Nathan P Gillett, Peter a Stott, and Kirsten Zickfeld (2009) "The Proportionality of Global Warming to Cumulative Carbon Emissions," Nature, Vol. 459, No. 7248, pp. 829-32.

Millar, Richard, Myles Allen, Joeri Rogelj, and Pierre Friedlingstein (2016) "The Cumulative Carbon Budget and its Implications," Oxford Review of Economic Policy, Vol. 32, No. 2, pp. 323-342.

van der Ploeg, Frederick (2018) "The Safe Carbon Budget," Climatic Change, Vol. 147 , No. 1 , pp. $47-59$.

Traeger, Christian (2014) "A 4-Stated DICE: Quantitatively Addressing Uncertainty Effects in Climate Change," Environmental and Resource Economics, Vol. 59, No. 1 , pp. $1-37$. 
Williams, Richard G, Philip Goodwin, Vassil M Roussenov, and Laurent Bopp (2016) "A framework to understand the transient climate response to emissions," Environmental Research Letters, Vol. 11, No. 1, p. 015003. 